\title{
Abstracts of the 2011 Paramedics Australasia Conference
}

\author{
Sydney, New South Wales, Australia \\ $7^{\text {th }}$ and $8^{\text {th }}$ October 2011
}

\section{Conference Presentation Abstracts}

\section{A model of paramedic decision making in cases involving pain. \\ Bill Lord}

\section{Monash University, Victoria, Australia.}

\section{Objectives}

Patients have a right to pain relief on humanitarian grounds. In addition, unrelieved pain may be associated with morbidity such as the development of chronic pain syndromes. Paramedics have an important role in relieving pain. However, therapeutic interventions that aim to reduce pain depend on clinical judgments that involve assessment of the of the patient's reported symptoms. As pain is a symptom that is difficult to objectively validate it is conceivable that clinician's beliefs and attitudes may modify clinical judgements and consequently influence decisions to withhold or initiate care. To investigate factors influencing paramedics' clinical judgments in cases involving pain the following research questions were developed: 1) What are paramedics' beliefs about pain and attitudes towards the assessment and management of pain in a community-based health setting; and 2) How could these beliefs and attitudes influence paramedics' clinical judgements in cases involving patients reporting pain.

\section{Methods}

Paramedics and student paramedics were invited to participate in focus group discussions that addressed pain and pain management in paramedic practice. Grounded Theory methodology was used to analyse transcripts of the focus group discussions, enabling the development of theories of decision making in the context of pain.

\footnotetext{
Results

Four major thematic categories emerged from the data: expressing pain, assessing the patient, believing the patient, and managing the patient with pain. Sub themes from each category were analysed to identify paramedic beliefs and attitudes that may influence clinical judgements. A model of clinical decision making was subsequently developed that uses an inputprocess-outcome taxonomy to illustrate variables
}

that may influence clinical decision making and subsequent actions when assessing patients with a complaint of pain.

\section{Conclusions}

This model of clinical decision making may help guide the education of paramedics to enable an awareness of factors that have the potential to adversely influence their clinical decision making.

\section{Epidemiology of acute heart failure presentations and prehospital treatment by paramedics in New South Wales, Australia: a retrospective analysis of over 7000 patients. \\ Paul Simpson ${ }^{1}$ and Jason Bendall ${ }^{2}$ \\ ${ }^{1}$ Ambulance Research Institute, Ambulance Service of New South Wales, Sydney, New South Wales, Australia \\ ${ }^{2}$ University of Sydney, Sydney, New South \\ Wales, Australia}

\section{Objective}

To describe the epidemiology of ambulance responses to patients with presumed acute cardiogenic pulmonary oedema (ACPO), their initial clinical presentation, and the impact of prehospital treatment on physiological vital signs.

\section{Methods}

A retrospective analytic epidemiological study was conducted into patients treated by paramedics for presumed ACPO between 1 July 2006 and 30 June 2008, utilising ambulance patient health care records (PHCR) linked with Medical Priority Dispatch System (MPDS) and Computer Aided Dispatch (CAD) data. Analysis was performed using SAS 9.2 to calculate descriptive statistics and differences between groups.

\section{Results}

There were 7,243 presentations included in the final analysis. The median age of patients was 81 years (IQR 74-87) and 53\% were female. Median scene time was 19 minutes (IQR 14-25) and total care time 31 minutes (IQR 24-40). Responses occurred most commonly between 0600 and 0900 hours $(19 \%)$ and during the colder, winter months. Seventy percent of patients received 
nitrates, $44 \%$ diuretics, and $22 \%$ morphine. The most common drug regime was nitrates alone (70\%), followed by nitrate/frusemide (19\%) and nitrate/frusemide/morphine (19\%). No drug therapy was administered in $18 \%$ of cases. Overall, there were statistically significant improvements in all vital signs, particularly systolic $\mathrm{BP}$ (mean 159 to $141 \mathrm{mmHg}$ ) and SpO2 (median $88 \%$ to $98 \%$ ).

\section{Conclusion}

ACPO is a common presentation with a distinct temporal pattern of presentation. Prehospital treatment appears to generally improve physiological variables; however its effect on longer term outcomes after leaving ambulance care is unclear and should be the subject of future research.

\section{A comparison of $10 \%$ and $50 \%$ dextrose in the management of prehospital hypoglycaemia: A before-and-after study James M Bews ${ }^{1}$, Bernard L Champion ${ }^{1,3}$, Jason C Bendall ${ }^{2,4}$ \\ ${ }^{1}$ University of Sydney, NSW, Australia \\ ${ }^{2}$ Ambulance Service of NSW, Rozelle, NSW, Australia \\ ${ }^{3}$ The Nepean Hospital, Kingswood, NSW, Australia \\ ${ }^{4}$ Ambulance Research Institute, Rozelle, NSW, Australia \\ Objectives \\ To compare the efficacy of $10 \%$ and $50 \%$ dextrose concentrations in the treatment of prehospital hypoglycaemia.}

\section{Methods}

This retrospective before-and-after study of patients with hypoglycaemia (blood glucose level $(B G L)<4 \mathrm{mmol} / \mathrm{L})$ was conducted in a large Australian ambulance service. Patients were administered either $10 \%$ dextrose or $50 \%$ dextrose. The main outcome measures were time taken for hypoglycaemia to be reversed, post treatment conscious level and post treatment blood glucose level (BGL).

Results: The study population comprised 2316 patients who met the inclusion criteria. There were no statistically significant differences between the two groups in baseline measures of patient age, gender, pre-treatment blood glucose level and pre-treatment GCS. Consciousness was restored faster following $50 \%$ dextrose than $10 \%$ dextrose $(p<0.001)$. Post treatment BGL was significantly lower in patients treated with $10 \%$ dextrose than in patients treated with $50 \%$ dextrose $(6.9 \mathrm{mmol} / \mathrm{L}$ versus $10.2 \mathrm{mmol} / \mathrm{L} p<0.001)$. The proportion of patients with a post-treatment BGL of $<6.7$ $\mathrm{mmol} / \mathrm{L}$ was higher following $10 \%$ dextrose when compared to $50 \%$ dextrose $(p<0.001)$ whereas the proportion of patients with a post-treatment BGL of $>11.1 \mathrm{mmol} / \mathrm{L}$ was higher following $50 \%$ dextrose when compared to $10 \%$ dextrose.

\section{Conclusions}

There appears to be no difference in the efficacy of $50 \%$ dextrose solutions compared to $10 \%$ dextrose solutions in restoring consciousness following hypoglycaemia. The time to restore consciousness was modestly faster with $50 \%$ dextrose than $10 \%$ dextrose and this may have operational benefits. A BGL below the optimal range was more frequent following $10 \%$ dextrose whereas a BGL above the optimal range was more frequent following $50 \%$ dextrose. It is unclear which dextrose solution is optimal for the prehospital management of hypoglycaemia. Irrespective of solution chosen, titration of the dextrose dose to within an 'optimal' range may improve clinical practice.

\section{Mathematical ability of first year undergraduate paramedic students - A before and after study Malcolm Boyle, Kathryn Eastwood, Nathan Stam}

\section{Monash University, Frankston, Victoria, Australia}

\section{Objectives}

An ability to accurately perform drug calculations unassisted is an essential skill for all health professionals, with various occupational-specific stressors exacerbating mathematical deficiencies. The scientific literature reveals practicing paramedics have alarmingly low levels of accuracy when performing unaided mathematical drug calculations. The objective of this study was to determine the unaided mathematic ability of first year undergraduate paramedic students before and after a mathematical and drug calculation program.

\section{Methods}

Students were administered a demographic, drug calculation and arithmetic questionnaire in the first week of semester and before starting the program to establish baseline performance. During the semester students participated in three tutorials which included both mathematical and drug calculation sessions without assistance of computational devices. The effectiveness of the program was assessed in a formative drug calculations examination at the end of semester. Descriptive statistics were used to describe the demographic and calculation answers with a paired t-test comparing the questionnaire and exam results. 


\section{Findings}

Conceptual, arithmetical, and computation ability was significantly improved following this education program and when comparing the correct results for the same question type $(n=63)$, there were statistically significant differences in four of five different drug calculations: volume of drug drawn up $10 \vee 57 p<0.0001$, infusion rate 29 v 31 $p=0.717$, drip rate $16 \vee 54 p<0.0001$, volume from a syringe $30 \vee 59 p<0.0001$, and drug dose $42 \mathrm{v}$ $62 p<0.0001$.

\section{Conclusions}

First year undergraduate paramedic students initially demonstrated a poor ability to complete mathematical and drug calculations without the assistance of computational devices on commencement of their study that improved significantly following appropriate education and practice. An ability to accurately and timely complete the mathematical and drug calculations is essential for paramedic practice and further study is required to determine the retention of this ability over time.

\section{Paramedic performed Fascia lliaca Compartment Nerve Block for Patients with Suspected Femoral Fractures: A paramedics personal perspective. Paul McRae ${ }^{1,2}$ and Jason C Bendall ${ }^{1,2}$ \\ ${ }^{1}$ Ambulance Research Institute, Ambulance Service of NSW, Rozelle, NSW, Australia ${ }^{2}$ Charles Sturt University, Bathurst, Australia}

\section{Objective}

This paper presents a paramedics experience in performing fascia iliaca compartment blocks (FICB), a form of regional anaesthetic nerve block, in the prehospital setting on adult patients with suspected or confirmed femoral fractures.

\section{Process}

Femoral (thigh) fracture is an important clinical problem and is associated with high morbidity and mortality, especially in the elderly. FICB has been performed successfully in both the inhospital and prehospital settings however there are no published reports of paramedic performed FICB. In the Ambulance Service of NSW a small cohort of paramedics have been trained to perform FICB in order to enrol patients into a prospective randomised controlled trial which is on-going (ACTRN12610000504011).

This paper will discuss pain management for femoral fractures, the FICB procedure and a personal perspective as one of the first intensive care paramedics to perform this procedure in the field. Enhancing paramedic scope of practice to include specific regional anaesthesia such as
FICB may ultimately benefit patients by minimising the unwanted systemic effects associated with conventional pain management protocols.

\section{Conclusion}

To date, the emergency medical community involved have shown great support for the trial, as they are witnessing firsthand the benefits patients are receiving from this intervention in their initial, but critical phase of care. The results of the prospective randomised controlled trial will better inform the role of paramedic performed FICB.

\section{Identification of out-of-hospital cardiac arrest in emergency calls in Victoria.} Janet Bray ${ }^{1,2}$, Jamie Walsh ${ }^{1}$, Tony Balm ${ }^{1}$, Karen Smith ${ }^{1,2}$

${ }^{1}$ Ambulance Victoria, Melbourne, Victoria, Australia

${ }^{2}$ Monash University, Melbourne, Victoria, Australia

\section{Objective}

Identification of out-of-hospital cardiac arrest (OHCA) in the emergency call is vital to the delivery of life-saving CPR instructions and rapid arrival of emergency medical services (EMS). The aim of this study was to examine rates of OHCA identification in metropolitan (computerised dispatch system) and rural (mostly noncomputerised dispatch system) regions of Victoria, and to examine its impact on known predictors of survival (bystander CPR and EMS response times).

\section{Methods}

The Victorian Ambulance Cardiac Arrest Registry (VACAR) was searched for EMS resuscitated adults cases of OHCA presumed to be of cardiac cause between 2003 and 2010. The proportion of these cases that were dispatched as a "cardiac arrest' was calculated and metropolitan and rural rates were compared. Also examined were differences in known predictors of survival.

\section{Results}

A total of 11029 OHCA were included: 8421 metropolitan and 2608 rural cases. Significant differences were seen the proportion of cases dispatched as 'cardiac arrest' between metropolitan and rural regions, $78 \%$ vs $43 \%$ respectively $(p<0.001)$; although rural dispatches were noted to increase over time, from 30\% identified in 2003-4 to $54 \%$ in 2008-10. The second most common dispatch type was the 'unconscious' event type (metropolitan 7\% and rural $30 \%$ ). In cases dispatched as 'cardiac arrest': bystander CPR rates were higher (metropolitan: $57 \%$ vs $27 \%, p<0.001$; and rural: $62 \%$ vs $47 \%, p<0.001)$, response times were 
shorter (metropolitan: 7.4 vs 8.4 minutes, $p<0.001$; and rural: 10 vs 11 minutes, $p=0.010$ ), and rates of VF/VT were higher (metropolitan: $41 \%$ vs $33 \%, p<0.001$; and rural: $42 \%$ vs $37 \%$, $\mathrm{p}=0.09)$.

\section{Conclusion}

The accurate dispatch of "cardiac arrest" is related to known predictors of survival in OHCA. The divide in OHCA between metropolitan and rural regions may be reduced with the introduction of the computerised dispatch system across the state in the next year.

\section{Out-of-hospital cardiac arrests in young adults in Melbourne, Australia - Adding coronial data to a cardiac arrest registry Conor Deasy, Janet Bray, Karen Smith, Stephen Bernard, Peter Cameron}

Department of Epidemiology and Preventive medicine, Monash University and Ambulance Vi

\section{Objective}

We aim to describe the coronial findings of young adults where the OHCA aetiology was 'presumed cardiac.'

\section{Methods}

Presumed cardiac aetiology OHCAs occurring in young adults aged 16-39 years were identified using the Victorian Ambulance Cardiac Arrest Registry (VACAR) and available coronial findings reviewed.

\section{Results}

We identified 841 young adult OHCAs where the Utstein aetiology was 'presumed cardiac'. Of these 740 died and 572 (77\%) OHCAs were matched to coroner's findings. On review of the coroner's cause of death, $230(40.2 \%)$ had a 'confirmed cardiac' aetiology, 221 (38.6\%) were proven 'non-cardiac', 97 (17\%) were inconclusive and $24(4.2 \%)$ cases remained 'open.' Confirmed cardiac' causes of OHCA were ischemic heart disease $(n=126,55 \%)$, cardiomegaly $(n=26$, $11.3 \%)$, cardiomyopathy $(n=25,11 \%)$, congenital heart disease $(n=15,6.5 \%)$, cardiac tamponade due to dissecting thoracic aorta aneurysm $(n=10$, $4.3 \%)$, myocarditis $(n=8,3.5 \%)$, arrhythmia $(n=7$, $3 \%)$, other $(n=13,5.7 \%)$. 'Non-cardiac' causes of OHCA were epilepsy/sudden unexplained death in epilepsy (SUDEP) $(n=56,25 \%)$, pulmonary embolism ( $n=29,13 \%)$, subarachnoid haemorrhage $(n=17,7.7 \%)$, other intracranial bleed $(n=7,3.2 \%)$, pneumonia $(n=17,7.7 \%)$, DKA $(n=16,7.2 \%)$, other complications of diabetes mellitus ( $n=8,3.6 \%$ ), complications of obesity $(n=9,4 \%)$, haemorrhage $(n=12,5.4 \%)$, sepsis $(n=8,3.6 \%)$, peritonitis $(n=6,2.7 \%)$, aspiration $(n=6,2.7 \%)$, renal failure $(n=5,2.3 \%)$, asthma $(n=5,2.3 \%)$ complications of anorexia $(n=3)$ and alcohol abuse $(n=2)$, thyrotoxicosis $(n=2)$, meningitis $(n=1)$ and others (12). Compared with coroner's diagnosed 'non-cardiac' OHCAs, 'confirmed cardiac' were more likely to be witnessed $(41 \% \vee 23 \%, p=<0.01)$, receive bystander CPR (35\% v 20\%, $p=<0.001)$, have a shockable rhythm $(27 \% \vee 6.3 \%, p<0.001)$ and have EMS attempted resuscitation (62\% versus $44 \%, p<0.001)$.

\section{Discussion}

Linking OHCA registries with coronial databases for aetiology of the arrest will improve the quality of the data and should be considered by all OHCA registries, particularly for young adult OHCA.

\section{Resuscitation of out-of-hospital cardiac arrests in residential aged care facilities in Melbourne, Australia. \\ Conor Deasy, Janet Bray, Karen Smith, Stephen Bernard, Peter Cameron}

Dept of Epidemiology and Preventive Medicine, Monash University and Ambulance Victoria, Melbourne, Australia

\section{Objective}

CPR in patients in residential aged care facilities (RACF) deserves careful consideration. We examined the characteristics, management and outcomes of out-of-hospital cardiac arrest (OHCA) in RACF patients in Melbourne, Australia.

\section{Methods}

The Victorian Ambulance Cardiac Arrest Registry (VACAR) was searched for all OHCAs occurring in RACFs in Melbourne. The characteristics and outcomes were compared to non-RACF patients in the VACAR.

\section{Results}

Between 2000 and 2009 there were 30,006 OHCAs, 2,350 (7.8\%) occurring in a RACF. A shockable rhythm was present in 179 (7.6\%) patients on arrival of paramedics of whom bystander CPR had been performed in $118(66 \%)$; $173(97 \%)$ received an EMS attempted resuscitation. ROSC was achieved in $71(41 \%)$ patients and $15(8.7 \%)$ patients survived to leave hospital. Non shockable rhythm was present in 2,171 patients $(92 \%)$ of whom $804(37 \%)$ had an attempted resuscitation by paramedics. ROSC was achieved in 176 patients $(22 \%)$ and 10 patients $(1.2 \%)$ were discharged alive. Survival from OHCA occurring in a RACF was less than survival in those aged $>70$ years of age who suffered OHCA in their own homes $(1.8 \% \vee 4.7 \%$, $\mathrm{p}=0.001$ ). On multivariable analysis, witnessed OHCA (OR 3.0, 95\% Cl 2.4-3.7) and presence of bystander CPR (OR 4.6, 95\% Cl 3.7-5.8) was associated with the paramedic decision to resuscitate. 


\section{Conclusion}

Resuscitation of patients in RACF is not futile. However, informed decisions concerning resuscitation status should be made by patients and their families on entry to a RACF. Where it is appropriate to perform resuscitation, outcomes may be improved by the provision of BLS training and possibly AED equipment to RACF staff.

\section{Out of hospital cardiac arrest outcomes - Is linkage to the National Death Index a viable option to determine survival rates? Judith Finn ${ }^{1,3}$, Karen Smith ${ }^{2}$, Ian Jacobs ${ }^{1,3}$ \\ ${ }^{1}$ The University of Western Australia, Perth, Western Australia, Australia \\ ${ }^{2}$ Ambulance Victoria, Melbourne, Victoria, Australia \\ ${ }^{3}$ St John Ambulance (Western Australia), Perth, Western Australia, Australia}

\section{Objectives}

Most Ambulance Services in Australia already have an out-of-hospital cardiac arrest (OHCA) registry. For those States without a welldeveloped population health data linkage system, determining long-term survival outcomes is pragmatically challenging. This study sought to ascertain the feasibility and accuracy of determining date of death through linkage to the Australian Institute of Health \& Welfare (AlHW) National Death Index (NDI).

\section{Methods}

Following AlHW ethics approval, the demographic details of all metropolitan OHCA patients transported to hospital by St John Ambulance (Western Australia) (or Ambulance Victoria) paramedics in 2009 were forwarded to AlHW for probabilistic linkage to the NDI. The accuracy of the best match date of death from the NDI was compared to the WA death records. (Analysis of the accuracy of linkage to Ambulance Victoria OHCA registry data is in progress.)

\section{Results}

Of the 375 WA cases, $314(83.7 \%)$ linked to a NDI death record. Of the 303 WA cases with both a WA death record and a NDI death record, the date of death was within 1 day difference for 294 $(97 \%)$ of cases. Of the 54 cases without a WA death record, 10 cases linked to a NDI death record, although most had flags identifying concerns about the linkage and 7 of the 10 patients had matching name and address listings in the current WA white pages telephone book. Of the 321 out of 375 WA cases with a WA death record, 18 did not link to a NDI record - however the fact that most of the missing deaths occurred within a 3 week period suggests a systematic error.

\section{Conclusions}

Whilst not 'perfect', linkage of state-based OHCA registry data to the AlHW NDI is a feasible option to determine survival outcomes in both the shortterm and long-term, especially for States without developed data linkage systems.

\section{Hospital characteristics are associated with patient outcomes following out-of- hospital cardiac arrest}

Dion Stub ${ }^{1,2}$, Karen Smith ${ }^{3,4}$, Janet Bray ${ }^{3,4}$, Stephen Bernard ${ }^{1,3}$, Stephen Duffy ${ }^{1,3}$, David Kaye $^{1,2}$

${ }^{1}$ Alfred Hospital, Melbourne Victoria, Australia, ${ }^{2}$ Baker IDI Heart \& Diabetes Institute, Melbourne Victoria, Australia

${ }^{3}$ Monash University, Melbourne Victoria,

Australia, ${ }^{4}$ Ambulance Victoria, Victoria, Australia

\section{Objective}

Post-resuscitation care may influence outcome following transport to hospital after resuscitation from out-of-hospital cardiac arrest (OHCA). We aimed to determine whether receiving hospital characteristics such as 24 hour cardiac catheterisation services, total bed number or OHCA patient volume influence the rate of survival.

\section{Methods}

We analyzed data from the Victorian Ambulance Cardiac Arrest Registry (VACAR) of patients from January 2003 to March 2010 who were transported to hospital with return of spontaneous circulation after OHCA.

\section{Results}

Ambulance paramedics attended 9971 patients with OHCA of suspected cardiac cause during the study period. Of these, $2902(29 \%)$ achieved return of spontaneous circulation and were transported to one of 70 hospitals. There were $1816(63 \%)$ treated at hospitals with 24 hour cardiac interventional services. After adjusting for differences in baseline characteristics, hospital factors significantly associated with survival were, treatment at hospitals with 24 hour cardiac interventional services (odds ratio $1.40 ; 95 \% \mathrm{Cl}$ 1.12-1.74, $p=0.003$ ) and patient reception between 0800 to 1700 (odds ratio $1.34 ; 95 \% \mathrm{Cl}$ $1.10-1.64, p=0.004)$. OHCA patient volume and total hospital bed number were not independently associated with outcome.

\section{Conclusion}

Hospital characteristics are associated with improved survival in patients with OHCA. This finding has implications for the establishment of 
regionalised systems of care for patients who have been resuscitated from OHCA.

\section{Long-term survival after resuscitation from out-of-hospital cardiac arrest.} Judith Finn ${ }^{1,2}$ and lan Jacobs ${ }^{1,2}$

${ }^{1}$ The University of Western Australia, Perth, Western Australia, Australia

${ }^{2}$ St John Ambulance (Western Australia), Perth, Western Australia, Australia

\section{Objectives}

To determine the long-term survival of out-ofhospital cardiac arrest (OHCA) patients who survived their initial arrest and identify factors associated with increased risk of subsequent death.

\section{Methods}

The Western Australian (WA) St John Ambulance cardiac arrest registry was used to identify all metropolitan OHCA patients discharged alive from hospital between 1996-2010. The study cohort was restricted to adults ( $\geq 18$ years) and persons resident in WA. The WA death register was used to identify the date of death up until 31 December 2010. The Kaplan-Meier method and log-rank test was used to compare the survival distributions of different sub-groups. Cox proportional hazards regression model was used to compute hazard ratios(HRs) for the association between total mortality and hypothesised prognostic factors, including: initial cardiac arrest rhythm, bystander CPR, aetiology and ambulance response intervals, with multivariable models adjusted for patient age, gender and calendar period.

\section{Results}

Between 1996-2010, 459 patients meeting the inclusion criteria were discharged from hospital alive: $74 \%$ males; mean age $62 \pm 15$ years; $38 \%$ paramedic witnessed; $37 \%$ bystander CPR; mean response (call-to-scene) $9.7 \pm 6.1 \mathrm{mins}$; $78 \%$ initial VF/VT and $91 \%$ of presumed cardiac aetiology. Of these 459 patients, $90 \%$ were alive at one year and $70 \%$ were alive at five years post arrest. The estimated median survival time from arrest to death was 11.7 years. The only significant predictor in the adjusted Cox regression model was age group. Whilst patients aged 75 years and over had a three-fold increase in the (adjusted) risk of death $(\mathrm{HR}=3.2,95 \% \mathrm{Cl} 2.1,4.6)$, almost half $(46 \%)$ of this older age group were still alive 5 years after their cardiac arrest.

\section{Conclusions}

Contrary to common misperceptions, the longterm prospects for OHCA survivors are far from bleak. Other than increasing age, there were no patient or arrest characteristics associated with a higher risk of death.

\section{Palliative Care for Mass Casualty Events with Scarce Resources}

\author{
Anne Wilkinson ${ }^{1}$, Maryanne Matzo ${ }^{2}$, Maria
}

Gatto $^{3}$, Joanne Lynn ${ }^{4}$, Sally Phillips ${ }^{5}$

${ }^{1}$ Edith Cowan University, Perth, Western

Australia, Australia

${ }^{2}$ University of Oklahoma Health Sciences

Center, Oklahoma City, Oklahoma, U.S.A.

${ }^{3}$ Bon Secours Health System, Marriotsville,

Maryland, U.S.A.

${ }^{4}$ Consultant, Washington, DC, U.S.A.

${ }^{5}$ Agency for Healthcare Research and Quality, Rockville, Maryland, USA

\section{Objectives}

Catastrophic mass casualty events (MCEs) can yield thousands of victims whose needs overwhelm local and regional health care systems, personnel, and resources. MCE response requires making difficult decisions regarding the allocation of scarce resources and the alteration of standards of care in all care settings. However, guidance regarding how to prepare for, manage, and provide medical care under such circumstances remains limited. Although the primary goal of a coordinated response to an MCE is to maximize the number of lives saved, a comprehensive response should also seek to minimize the suffering of those who may not survive. The purpose of this paper is to explore the role of palliative care in the support of individuals not expected to survive an MCE and to provide recommendations for specific actions for a coordinated disaster response plan.

\section{Methods}

Semi-structured telephone discussions with disaster management experts and an expert group meeting identified issues, roles, responsibilities, procedures, and resources offering benefits of integrating palliative care into disaster planning and response.

\section{Results}

The investigations identified five domains of concern, along with guidance: (1) the role of palliative care in a mass casualty event with resulting scarce resources; (2) the triage and ensuing treatment decisions for those "likely to die;" (3) the critical palliative care services to provide, along with the personnel and settings; (4) the pragmatic plans needed for ensuring training, supplies, and organizational or jurisdictional arrangements; and (5) unusual issues affecting palliative care under MCE scenarios.

\section{Conclusions}

Palliative care minimizes the suffering of those who die, ensures comfort, addresses the needs of those who will not survive, and may also free up resources to optimize survival of others. 
Planning to provide palliative care during mass casualty events should be part of the current state and local disaster planning/training guidelines, protocols, and activities.

\section{Perceptions of clinical leadership in St. John Ambulance Service WA: A research report}

David Stanley and Joseph Cuthbertson

Curtain, Perth, WA, Australia

\section{Objectives}

To identify how clinical leadership is perceived by paramedics and ambulance personal in the course of their everyday work and the effectiveness and consequences of the application of clinical leadership in pre-hospital care delivery.

\section{Methods}

A questionnaire was distributed via in-service training sessions to St. John Ambulance operational staff in WA between February 2010 and November $2010(n=250)$. The methodological principals of the study were based on phenomenology. Analysis of the quantitative data was via SPSS software and the qualitative data was analysed by spreadsheet and word documents.

\section{Results}

Of the 250 ambulance service staff who attended in-service education between February 2010 and November 2010, 104 returned questionnaires, a return rate of $41.6 \%$. Of those respondents, their average length of service with the St. John Ambulance Service was just under 7 years (6.9 years), with the longest service of any respondent being 30 years.

Most respondents recognised that clinical leaders were involved in team work, the generation of new ideas, effective communication and involved others appropriately. While clinical experience was valued highly, research skills or qualifications were less well recognised as an aspect of a clinical leader. What mattered was that the values of the clinical leaders were matched by their actions and abilities.

\section{Conclusion}

It is hoped that with a better understanding clinical leadership and how it is perceived by paramedics and ambulance officers they will be able to play a more effective part in service improvement, the implementation of a quality agenda and impact positively on pre-hospital care delivery. A better understanding of clinical leadership may support a more responsive and effective ambulance service where the focus can remain on the clinical aspects of the paramedic's role.

\author{
Paramedic Response to Suicide \\ Bombings: Learning from the Israeli \\ Experience \\ Christopher R. Foerster ${ }^{1,2}$ and Aaron Richman ${ }^{3}$ \\ ${ }^{1}$ James Cook University, Townsville, \\ Queensland, Australia \\ ${ }^{2}$ Lambton EMS, Lambton County, Ontario, \\ Canada \\ ${ }^{3}$ Philadelphia University, Philadelphia, PA, USA
}

\section{Objective}

Suicide bombings have been seen all around the world, with particularly high numbers in Israel.

Paramedics are an integral part of the response to these bombing incidents. Most countries have not responded to a suicide bombing and without this experience, the response to their first incident is likely be suboptimal. By examining what the Israeli paramedics have learned in their experience with suicide bombing, paramedics in other areas can be better prepared if they ever need to respond. The objective of this research was to determine the important components of the Israeli Emergency Medical Services (EMS) response to suicide bombings that allow for their success.

\section{Methods}

Reviews of open source information were used as well as visits to multiple suicide bombing sites in Israel with reviews of these bombing incidents and their responses. These were analysed for common themes important to effective suicide bombing responses.

\section{Results}

It is essential that the first arriving ambulance crew be fully capable of initialising the medical response to the incident. While doing so, they must maintain a strong situational awareness, considering both secondary devices and confirmation of neutralisation of known threats. The Israeli approach include immediate initiation of command and triage with patients being evacuated from the scene quickly. Coordination between front-line commanders is even more important at these incidents. Practising a scaled response and working to establish a collective knowledge as well as joint training is important in preparing for these types of responses.

\section{Conclusions}

Suicide bombings are unique incidents that will challenge any EMS system, particularly if they are not experienced in suicide bombing response. Knowledge of these unique response considerations is essential for paramedics to be able to respond safely and effectively to these incidents. 
The influence of research on the policy and practice of ambulance services in the United Kingdom: Lessons for Ambulance Authorities and Paramedics Peter O'Meara ${ }^{1,3}$ and Malcolm Woollard ${ }^{3,2}$

${ }^{1}$ La Trobe University, Bendigo, Victoria, Australia

${ }^{2}$ Charles Sturt University, Bathurst, NSW, Australia

${ }^{3}$ Coventry University, Coventry, Midlands, UK

\section{Background}

Ambulance services recognise the importance and value of using evidence in their decisionmaking. This represents a shift in process that is challenging to implement given the paucity of robust evidence to support pre-hospital interventions and the dearth of expertise in interpreting research findings.

\section{Objective}

This study examined how and to what extent policy makers in United Kingdom Ambulance Trusts use evidence to inform their decisionmaking.

\section{Methods}

Data was collected and analysed using a 'process model' of evidence utilisation to examine the translation of research into policy and practice. Semi-structured interviews were conducted with established academic researchers in paramedic practice / ambulance systems, members of the College of Paramedics, and key decision makers within each of the participating Trusts.

\section{Results}

Decision making in UK Ambulance Trusts is partly driven by evidence. Researchers see varying levels of research engagement and turning copious data into good research is a challenge. Trusts have appointed research leads and formed a collaborative National Ambulance Research Steering Group. However, there remain few research educated paramedics and managers who are ready to embrace evidence based practice.

\section{Conclusions}

One of the major challenges is how ambulance services and researchers manage their relationships when they may have very different views of the world, with many researchers allegedly placing 'evidence' above other considerations such as public confidence [and organisational image that managers are required to consider when making policy decisions. This clash of values revolves around their respective concepts of what evidence is and the importance they attach to the influence of context in the decision-making processes.

\section{Disaster preparedness in small communities with a known threat. Mieke Couling}

\section{AUT University, Auckland, New Zealand}

\section{Background}

Small communities lack many of the advantages of larger cities when it comes to emergency management support. However, historically they have held a reputation for being self-sufficient and resilient in the face of adverse situations. Despite this image of self-sufficiency and resilience, small communities with a known hazard are not necessarily better prepared or informed than residents of larger cities ${ }^{1}$. The isolated East Coast communities of the Coromandel Peninsula in New Zealand are at high risk of tsunamis ${ }^{2}$. In September 2009 a complete evacuation of the town Pauanui was undertaken following the Samoan Tsunami.

\section{Objectives}

This study aims to establish how well prepared people in Coromandel are for a Tsunami by exploring the knowledge, understanding and feelings about disaster preparedness of people living in this small community.

\section{Method}

Qualitative data exploring people's reactions and knowledge was collected from fifteen semistructured interviews of evacuated residents of the Coromandel. The interviews were thematically analysed and revealed common themes and gaps where the emergency management systems were deficient. The actions taken by the First Responders (police, fire and ambulance) were included in the analysis

\section{Results}

Overall, this study found gaps in the residents' knowledge of the hazards facing their community and found a concerning lack of preparation for quick evacuation. The study also highlighted that people have a high reliance on official warning systems and struggle to interpret natural warning signals such as earthquakes.

\section{Conclusion}

If emergency services appreciate the strengths and weaknesses of preparedness in small communities that are at risk of a particular threat or hazard, they can better prepare their own response plans and contribute more effectively to the community plans and education. 


\section{A model for the management and leadership of volunteer emergency health providers}

Peter O'Meara' ${ }^{1}$, John Rae ${ }^{2}$, Vianne Tourle ${ }^{2}$

${ }^{1}$ La Trobe University, Bendigo, Australia,

${ }^{2}$ Charles Sturt University, Bathurst, Australia

\section{Background}

Ambulance services operating in rural and remote areas are highly reliant on volunteer and first responder models of service delivery. Despite the importance of this volunteer workforce, little has been reported on the management of these emergency health providers.

\section{Objective}

To identify those factors that may facilitate the successful integration of volunteer emergency health providers into ambulance services and make recommendations highlighting good management practice.

\section{Methods \\ Senior executives of ambulance services from one region of New Zealand and seven states of Australia were surveyed and interviewed. They were asked to describe their ambulance service's approach to volunteers and first responders. Three researchers analysed these data using classic content analysis techniques and computer- assisted programs - NVIVO and Leximancer to develop a volunteer emergency health provider management model.}

\section{Findings}

Analysis of interview transcripts and publicly available data revealed facilitative factors associated with strong, vibrant ambulance volunteer systems. These facilitative factors are: commitment to the volunteer model of service delivery; a degree of management decentralization and volunteer input into decisionmaking; commitment of resources toward the volunteer model; and the organizational integration of volunteers into the ambulance service. These facilitative factors informed a volunteer emergency health provider management model which consists of four components: leadership; integrative processes; resource commitment within the lead organisation; and the relative autonomy of the volunteers.

\section{Conclusions}

This model aims to encourage the adoption of positive and innovative strategies to improve the integration of ambulance volunteers and first responders in ambulance services. If elements of the model were replicated more widely, a viable and effective volunteer emergency health response system could be established in those areas where it is uneconomic or impractical to provide a salaried ambulance service staffed with professionally qualified paramedics.

\section{Peer-assisted teaching and learning in paramedic education: Preliminary findings Michael Fox, Christian Winship, Whitney Williams, Sinead Leaf, Brett Williams, Leanne Boyd}

\section{Monash University, Melbourne, Australia}

\section{Objectives}

Peer-assisted teaching is growing internationally as a beneficial pedagogical strategy in health professional education. Paramedics are continually engaged in teaching students, patients and their families - so these developments are highly relevant to our discipline. This project seeks to explore: i) third year students' experiences of teaching and assessing junior students, and ii) first year students' experiences of being taught and assessed by senior students.

\section{Method}

An investigation of peer-assisted teaching and learning among first and third years at Monash University was investigated using the Peer Teaching Experience Questionnaire (14 items) and the Clinical Teaching Preference Questionnaire (11 items). Both self-reporting measures used a 5 -point Likert scale ( $1=$ Strongly Agree and 5=Strongly Disagree).

\section{Results}

A total of 154 students participated in the study: $n=127$ first years $(n=87$ control group, $n=40$ intervention group), and $n=27$ third years $(n=8$ control group and $n=19$ intervention group). The majority of students were $<26$ years of age $n=130$ $(84 \%)$ and female $n=100$ (63\%). Ninety-four percent $(n=120)$ first year students felt (strongly agree or agree) 'teaching is an important role for paramedics'. Almost two-thirds $n=82(64 \%)$ felt (strongly agree or agree) 'being taught by peers increased their collaboration with other students compared with their instructor'. There were several items that were statistically significant $(p<0.001)$ between the first years: 'I am less anxious when performing a paramedic skill in the presence of my peers', and 'I can communicate more freely with my peers than with my instructor'. All third year students $n=27(100 \%)$ 'felt teaching is an important role for paramedics', and that 'there should be more opportunities for peer teaching in the curriculum'

\section{Conclusion}

Results from this study support a larger scale study in the future. Preliminary results support the utility of peer-assisted teaching and learning. Consideration should be given for inclusion into existing paramedic curricula nationally. 


\section{Can relief from chest pain with nitrates inform a clinical diagnosis?}

Laura Roberts, Lynsey Smit, Ziad Nehme, Brett Williams

\section{Monash University, Melbourne, Australia}

\section{Objectives}

The administration of nitrates in the setting of ischaemic chest pain has been a mainstay of paramedic practice for many years. Its longstanding use has led to the belief that chest pain eased by nitrates is predictive or diagnostic of active coronary artery disease. To examine this assumption, we conducted a review of the literature to determine whether chest pain relief with nitrates can predict a diagnosis of underlying cardiac aetiology.

\section{Methods \\ A literature review was undertaken using the electronic databases Ovid Medline, CINAHL Plus and the Cochrane Library from their \\ commencement to the end of May 2011. \\ Keywords used in the search included: \\ [Myocardial Ischaemia or "chest pain" or "angina"] and ["anginine" or "glyceryl" or "nitrate" or "nitroglycerin" or "NTG" or "GTN"]. Studies were eligible for inclusion if they reported the effect and diagnostic accuracy of nitrate use for predicting underlying active coronary artery disease.}

\section{Findings}

Of the initial 608 articles, 38 potential studies were identified after screening title and abstract. From these, 4 studies met the eligibility criteria. Considerable heterogeneity was identified in the prevalence of disease, description of the reference standard, and definition of 'response to nitrates'. No study was blinded. The predictive value and likelihood ratios of chest pain relief by nitrates were generally poor. Sensitivity varied considerably between studies ( 0.35 to 0.92 ), however all reported low specificity $(0.12$ to 0.52$)$. In all studies the positive and negative likelihood ratios closely approximated 1.0

\section{Conclusion}

While nitrates may be clinically indicated to reduce chest pain in patients with angina, there is no empirical evidence to support its use as a predictor of active coronary artery disease. Thus, relief from chest pain with nitrate use does not indicate nor exclude underlying cardiac aetiology and should not be used to inform a clinical diagnosis.

\section{Assessment of the Glasgow Coma Scale: A pilot study examining the accuracy of paramedic undergraduates. \\ Christian Winship, Brett Williams, Malcolm Boyle}

\section{Monash University, Melbourne, Australia}

\section{Objectives}

Accurate assessment of a patient's conscious state using the Glasgow Coma Scale (GCS) is an important skill for paramedics as it may determine the patient's initial and ongoing management, including advanced airway management and synchronised cardioversion. To our knowledge, there are no previous studies that have reported the accuracy of undergraduate paramedics' interpretations of a patient's conscious state using the GCS. Therefore, the objective of this study was to determine if undergraduate paramedic students from an Australian University were able to accurately interpret a variety of conscious states using a simulated patient.

\section{Methods}

A prospective single-blinded observational pilot study requiring students to interpret the conscious state of four adult patients using the GCS by viewing a simulation DVD package.

\section{Results}

There were 137 students that participated in the study, of which $65 \%(n=87)$ were female. The results demonstrated that undergraduate paramedic students were unable to accurately interpret a range of patient conscious states with only $20 \%$ and $37 \%$ of participants able to accurately identify the GCS of patients 2 $(\mathrm{GCS}=12)$ and $3(\mathrm{GCS}=7)$. For patient 2 there was a statistically significant difference between the BN/BEH students and the BEH year one $(p<0.0001)$ and year two $(p=0.022)$ students. The motor component of the GCS appeared to be the component where the least accurate interpretation occurred, with only $47 \%$ of students being able to accurately identify the criteria that patient 3 displayed. Participants were however able to accurately interpret the GCS of both patient 1 $(\mathrm{GCS}=14)(86 \%)$ and patient $4(\mathrm{GCS}=15)(92 \%)$.

\section{Conclusion}

This study demonstrates that undergraduate paramedic students from an Australian university are unable to accurately interpret a patient's conscious state if their GCS score was $<14$. These findings have provided staff with important data for considering alternative teaching and learning approaches in conscious state assessment. 


\section{Is the standard $4.5 \mathrm{~cm}$ cannula long enough for a needle thoracostomy? Chloe Abel and Malcolm Boyle}

\section{Monash University, Frankston, Victoria, Australia}

\section{Objectives}

Tension pneumothorax is a life threatening condition which is treated in the pre-hospital setting by needle thoracostomy. Studies have shown that the needles commonly used to be of inadequate length to penetrate the pleural cavity. The objective of the study was to identify if chest wall thickness and/or catheter length were a reason for failed prehospital needle thoracostomy.

\section{Methods}

A literature search was conducted using medical electronic databases, MEDLINE CINHAL, EMBASE, and Cochrane Central Register of Controlled Trials (CENTRAL). These databases were searched from January 1996 until the end of March 2011. References from articles retrieved were reviewed. Search keywords included prehospital, EMS, EMT, chest wall thickness, tension pneumothorax, pneumothorax, thoracostomy, needle thoracostomy, and decompression. Articles were included if they reported the prehospital use of needle thoracostomy and failure rates relating to needle length and chest wall thickness. Non-English and hospital based articles were excluded.

\section{Results}

There were 3,828 articles located by the search with six articles meeting the inclusion criteria. The mean chest wall thickness at the second intercostal space mid clavicular line varied between $2.2 \mathrm{~cm}$ and $5.36 \mathrm{~cm}$, with the thickest chest wall being $9.35 \mathrm{~cm}$. A variation between left and right side chest wall thickness also exists. One study identified a $30 \%$ increase in females and $18 \%$ increase in males for arms by the side compared to raised above the head. The majority of studies used the $4.5 \mathrm{~cm}$ catheter with one study using a $5 \mathrm{~cm}$ catheter and another a $3.2 \mathrm{~cm}$ catheter. Failure rates varied from $81 \%$ with the $3.2 \mathrm{~cm}$ catheter to $23 \%$ with the $5 \mathrm{~cm}$ catheter. The failure rate for the $4.5 \mathrm{~cm}$ catheter ranged from $10 \%$ to $50 \%$.

\section{Conclusions}

The study findings suggest a large proportion of the population have chest walls thicker than the standard $4.5 \mathrm{~cm}$ catheter and that larger catheters may be warranted.

\section{Occupational Risks on Undergraduate Paramedic Clinical Placements \\ Tegwyn Bath, Jade Sheen, Leanne Boyd}

Monash University, Frankston, VIC, Australia

\section{Background}

Clinical placements are a vital part of undergraduate prehospital education, aiming to provide 'on the job' skill acquisition and link theory with practice. A number of occupational risks may occur during these clinical placements, potentially putting in jeopardy student learning opportunities as well as student health and wellbeing. Clinical placements have been identified as having a strong impact on students career plans, and it is vital that safe learning environments are provided for students.

\section{Objectives}

- To examine the nature and context of occupational risks encountered by undergraduate students on paramedic clinical placements

- To gain an understanding of risk management strategies either in place or required to minimise the consequences and impact of occupational risks on students.

\section{Methods}

Firstly, a literature review was undertaken to identify literature related to occupational risk in the clinical placement and prehospital settings. Secondly, a cross sectional study using paperbased questionnaires was undertaken by undergraduate students who had undertaken a minimum of four days of paramedic clinical placement. Thirdly, a series of focus groups were held to allow in-depth exploration of the nature and context of student experiences of occupational risk on clinical placement. Ethics approval was granted.

\section{Findings}

Initial findings (final data to be collected June 2011 and analysed by the time of the Conference) indicate that undergraduate students encounter a range of occupational risks while on paramedic clinical placements. Key themes include workplace injury, occupational violence, stress, bullying and harassment. The incidence of bullying and covert harassment by qualified paramedics towards undergraduate students under their supervision was of particular concern. Students demonstrated an awareness of the physical and psychological risks associated with this part of their training, but a lack of interest in incident reporting. Of the students that reported physical or psychological injury occurring on clinical placement, few sought help. 


\section{Australian EMS in an international comparison: a social media experiment Florian Breitenbach}

\section{Edith Cowan University, Perth, Western \\ Australia, Australia}

\section{Objective}

To compare Australian EMS systems and their environment with that of the UK \& the US

\section{Methods \\ Professionals from three countries (England, Scotland and the United States) were contacted via their online presence (blog and/or twitter account), via personal recommendation and chance encounter. All interviewees were paramedics in their system, additional qualifications included some being supervisors, university lecturers, clinical educators and film makers. All paramedics were interviewed on a set list of questions regarding their subjective experience of working in their area and country. Additional objective information was obtained from clinical guidelines and laws from the respective services, states and countries.}

\section{Conclusion}

Australian EMS systems and structures don't need to shy away from an international comparison, and are leading on many levels. Working conditions, paramedic autonomy, and especially the widespread university-led education are amongst the factors that puts Australia in an excellent position in an international comparison.

\section{Impact of paramedic and patient gender on prehospital pain management}

\section{Bill Lord ${ }^{1}$, Jason C Bendall ${ }^{2,3}$, Tracie Reinten-} Reynolds ${ }^{2,4}$

${ }^{1}$ Department of Community Emergency Health and paramedic Practice, Monash University, Melbourne, VIC, Australia

${ }^{2}$ Ambulance Research Institute, Rozelle, NSW, Australia

${ }^{3}$ Ambulance Service of NSW, Rozelle, NSW, Australia

${ }^{4}$ NSW Biostatistical Officer Training Program, NSW Health, North Sydney, NSW, Australia

\section{Objective}

To better understand the influence of patient gender and paramedic gender on analgesic administration in the prehospital setting.

\section{Methods}

A retrospective study of analgesic administration in lucid adult patients (16-100 years old) with moderate to severe pain (verbal numerical rating score 4-10) by paramedics authorised to administer opiate and non-opiate analgesia was
}

undertaken. Data was obtained from dispatch, clinical and workforce data. The study was approved by the Monash University Human Research Ethics Committee. Analysis was undertaken using logistic regression techniques. Differences were considered significantly different when $\mathrm{p}<0.05$.

\section{Results}

The study population comprised 42,051 patients, $51 \%$ of which were administered an analgesic agent. The median age of patients was 57 years (IQR $38-75$ ) and $50.4 \%$ were female. Pain management was provided by 1,255 paramedics of whom $19 \%(n=239)$ were female. A female paramedic provided treatment for $13 \%$ of patients receiving analgesia. For the outcome of receiving any analgesia, neither patient gender nor paramedic gender was predictive $(p=N S)$. In a multivariate model for the outcome of receiving any analgesia, patient gender, paramedic gender and the interaction between patient and paramedic gender were all statistically nonsignificant $(p=N S)$. For the outcome of receiving opiate analgesia (i.e. morphine or fentanyl), male patients were at greater odds of receiving an opiate (OR 1.28 95\% Cl 1.23-1.33 p<0.0001). Paramedic gender was not predictive of whether an opiate was given $(p=N S)$. In a multivariate model for the outcome of receiving opiate analgesia, whilst patient gender remained significant $(p<0.0001)$, paramedic gender and the interaction between patient and paramedic gender remained statistically non-significant $(p=N S)$.

\section{Conclusion}

Paramedic gender does not appear to influence the administration of analgesia in this study. Male patients are more likely to receive opiate analgesia. This finding is not explained by paramedic gender.

\section{Do younger women have better OHCA survival rates than men? - Exploring the "oestrogen effect". Janet Bray $^{1,2}$, Dion Stub ${ }^{2,3}$, Stephen Bernard ${ }^{1}$ , 2 , Karen Smith ${ }^{1,2}$ \\ ${ }^{1}$ Ambulance Victoria, Melbourne, Victoria, Australia ${ }^{2}$ Monash University, Melbourne, Victoria, Australia \\ ${ }^{3}$ Baker IDI Heart Diabetes Institute, Melbourne, Victoria, Australia}

\section{Objective}

Recent studies have suggested women of a childbearing age have better cardiac arrest outcomes than men of a similar age. We aimed to investigate this finding using the Victorian Ambulance Cardiac Arrest Registry (VACAR) in a 
group of non-traumatic out-of-hospital cardiac arrests (OHCA).

Methods
VACAR was searched for OHCA between 2003 and 2010 meeting the following inclusions criteria: 1) EMS resuscitated, 2) presumed cardiac aetiology, 3) arrest was not witnessed by paramedics, and 4) aged 18-44 years. Statistical differences between men and women were assessed, including differences in the known predictors or survival and logistic regression survival analysis.

\section{Results}

For the study period, there were $921 \mathrm{OHCA}$ meeting inclusion criteria; 282 females and 639 males (representing $10 \%$ of male OCA and $10 \%$ of female OHCA). Differences were seen in the known predictors of survival between males and females. Females were slightly younger (38 vs 39 years, $p=0.01$ ) and less likely to: arrest in a public location ( $15 \%$ vs. $36 \%, p<0.001)$, have a witnessed arrest ( $46 \%$ vs. $58 \%, p<0.001)$, receive bystander CPR ( $56 \%$ vs. $63 \%, p=0.06)$; have an initial shockable rhythm (35\% vs. $47 \%, p=0.002)$. Similar rates of survival to hospital discharge were seen between males and females both overall (16\% vs $14 \%, p=0.47)$ and for shockable rhythms (30vs 31, $p=0.80$ ). In multivariate analysis, adjusting for known predictors of survival, gender was not an independent predictor of survival (OR 1.24, 95\% Cl: 0.77-2.02).

\section{Conclusion}

Although young women are less likely to have factors linked to survival -they have similar survival rates to young men. Though this may suggest some impact of gender on survival, gender was not independently associated with survival. Therefore, our findings do not support evidence from in-hospital studies demonstrating increased survival in women of a child bearing age.

\section{Are we under-resuscitating our children? A review of paediatric age-based weight estimation formulae for the western population. Ziad Nehme}

Monash University, Melbourne, Victoria, Australia

\section{Objectives}

For almost 20 years, the Advanced Paediatric Life Support (APLS) weight estimation formula has been widely adopted to estimate paediatric weights in emergency settings. In light of the increasing weight of children in Australia, we conducted a review of contemporary age-based weight estimation formulae for a western population of children.

\section{Methods \\ The Ovid Medline database was searched utilising a simple search strategy with the search terms ["paediatric" or "children"] and "weight" and ["estimation" or "formula" or "calculate" or "equation"] from 1996 to 9th March 2011. A highly selective eligibility criterion was devised to extract articles that were considered relevant to the research objective: 1) Recruitment of patients post-2000; 2) Recruitment within the emergency department or acute care setting; 3) Patients aged at least 1 to 11 years inclusive; 4) Derived in a select western population, and; 5) Reported the derivation or validation of an age-based linear formula.}

\section{Results}

This review identified two new paediatric weight estimation formulae that are more accurate than the current APLS formula. Both the Luscombe and Best Guess methods have been derived and validated in a large western population of children. A mean percentage error of $+4.2 \%$ for infants, $+2.6 \%$ for the preschool-aged, and $+7.7 \%$ for school-aged children was observed with the Best Guess formula. The proportion of Best Guess weights within $20 \%$ of the recorded weight was as high as $83 \%$ in preschool and $60 \%$ in school-age children. The performance of the Luscombe formula was similar to the Best Guess and better than the APLS formula.

\section{Conclusions}

The Australian Best Guess formula offers a locally-derived and validated method of agebased weight estimation for children that is more accurate than the APLS formula. While the Luscombe formula offers similar accuracy, it is yet to be validated in a population of critically ill patients.

\section{Oxygen therapy for acute stroke: Is it time to rethink our approach?}

Stuart Howard, Anthony Mead, Chloe-Eloise Verhees, Ziad Nehme, Brett Williams

\section{Monash University, Melbourne, Australia}

\section{Objective}

The administration of routine oxygen therapy for acute stroke remains widespread in prehospital care. Recently, the National Stroke Foundation revised acute stroke guidelines to recommend a shift away from the use of oxygen therapy in nonhypoxic patients. In light of this change, we sought to appraise the evidence-based literature to identify the effect of intervention for all acute stroke patients receiving normobaric oxygen therapy. 


\section{Methods}

A literature review was undertaken utilising the electronic databases Ovid Medline, EMBASE, and CINAHL from their commencement to the end of May 2011. Keywords used in the search included: ["stroke" or "cerebrovascular" or "CVA"] and "oxygen". Clinical trials were included if they provided measurable clinical outcomes for acute stroke patients randomised to receive normobaric oxygen therapy or room air within 24 hours of symptom onset. Eligible studies were limited to those in English-language and involving human participants.

\section{Findings}

The search strategy identified 90 potential studies, of which 4 clinical trials met the eligibility criteria. Short-term functional outcomes were improved in patients randomised to oxygen therapy. An improvement in stroke scale scores up to 3 months follow up was observed in one study $(p=0.03)$, while another reported the benefits of neurological recovery with oxygen therapy to be as limited as 1 week (OR 2.9; 95\% Cl 1.59 to 5.4). One large quasi-randomised trial reported a significant reduction in death for mild to moderate strokes randomised to room air at 7 months follow-up (OR $0.45 ; 95 \% \mathrm{Cl} 0.23$ to 0.90 ).

\section{Conclusion}

The benefits of oxygen therapy in stroke patients appear short-term, with improvements in neurological recovery limited to 1 week. Long term outcomes show an increase in death for select patients receiving routine oxygen therapy. Until further studies can demonstrate its safety, the routine use of oxygen therapy in stroke cannot be recommended.

\section{Paramedics \& "psych" patients: What we see \& hear, our actions \& our rationale. An ethnographic study exploring paramedic culture, understanding, beliefs and actions when attending psychiatric presentations. \\ Louise Roberts}

\section{Flinders University, Adelaide South Australia, Australia}

\section{Objective}

Paramedics are currently in a world where their role and scope of practice is rapidly changing. They are in a position where the boundaries are being repositioned and they find themselves confronted by situations that require extended care and patients that do not fit the traditional mould of emergency care. The research question for this doctoral study asks how in this changing environment do paramedics identify, assess and manage psychiatric presentations in the community. The change from institutional to community based care in mental health reform has seen increases in emergency department attendance of individuals needing mental health care. A large majority of these individuals are attended to and transported by paramedics. As the link between pre-hospital and further care paramedic have a vital role to play, but to date paramedics' 'on-road' experiences and culture surrounding what they do when attending psychiatric presentation had not been widely researched.

\section{Methods}

This ethnographic study was conducted in 2009 to early 2010 and involved observation of a tertiary hospital emergency department and the ambulance arrival area over an eleven month period. It included a series of twenty unstructured interviews and informal conversations with paramedics and document analysis of the Ambulance Patient Report Form. The ethnographic method allowed paramedics to openly communicate how they viewed their practice and actions with patients they considered psychiatric presentations.

\section{Results}

This paper focuses on the themes of compliance and non-compliance, caution and risk and the role that knowledge and paramedics' perception of their work plays in their approach and assessment.

\section{Conclusion}

Understanding the organisational, social and contextual nature of paramedic work with patients suffering a mental illness will assist future developments in education, policy and service provision by paramedics.

\section{Ketamine is superior to morphine alone for the management of traumatic pain in the prehospital setting: A randomized controlled trial}

\author{
Paul Jennings ${ }^{1,2}$, Peter Cameron ${ }^{1,3}$, Stephen \\ Bernard ${ }^{1,2}$, Tony Walker ${ }^{2}$, Mark Fitzgerald ${ }^{3}$, \\ Kevin Masci $^{2}$
}

${ }^{1}$ Monash University - Department of Epidemiology and Preventive Medicine, Melbourne, Australia

${ }^{2}$ Ambulance Victoria, Melbourne, Australia

${ }^{3}$ The Alfred Hospital, Melbourne, Australia

\section{Objectives}

To assess the efficacy of intravenous (IV) ketamine compared to IV morphine in reducing pain in adults with significant pre-hospital traumatic pain despite initial treatment with $5 \mathrm{mg}$ IV morphine. We also aimed to assess the safety 
of ketamine compared to morphine by comparing changes in vital signs and conscious state, and the incidence of side effects including, excessive sedation, significant hypotension or hypertension, arrhythmia, nausea and emesis.

\section{Methods}

This study was a prehospital, prospective, randomized, controlled, open-label study. Patients with trauma and a verbal pain score of $>5$ after $5 \mathrm{mg}$ IV morphine were eligible for enrolment. Patients allocated to ketamine received a bolus of $20 \mathrm{mg}$ followed by $10 \mathrm{mg}$ every 3 minutes. Patients allocated to morphine alone received $5 \mathrm{mg}$ IV every 5 minutes until pain free. Pain scores were measured at baseline and at hospital arrival.

\section{Results}

A total of 135 patients were enrolled between December 2007 and July 2010. There were no differences between the groups at baseline. Following the initial $5 \mathrm{mg}$ dose of IV morphine, patients allocated to ketamine received a mean of $40.6(+/-25) \mathrm{mg}$. Patients allocated to morphine alone received a mean of $14.4(+/-9.4) \mathrm{mg}$. There mean pain score change was $-5.6(95 \% \mathrm{Cl}-6.2$ to $-5.0)$ in the ketamine group compared with -3.2 $(95 \%-3.7$ to -2.7$)$ in the IV morphine group $(p<0.0001)$. The IV morphine group had $9 / 65$ (14\%) side effects reported compared with $27 / 70$ $(39 \%)$ side effects in the ketamine group $(P=0.001)$.

\section{Conclusion}

The use of ketamine significantly decreases prehospital pain scores compared with morphine alone in adult patients with moderate to severe traumatic pain but is associated with an increase in the rate of minor side-effects.

\section{Evaluating the effects of exposure to trauma for paramedics and pre-hospital workers in rural and remote Western \\ Australia}

Joseph Cuthbertson ${ }^{1}$, Allyson Browne ${ }^{2}$,

Stephan Shug ${ }^{3}$, Elizabeth Newnhman ${ }^{4}$

${ }^{1}$ St John Ambulance WA Inc., WA, Australia

${ }^{2}$ Statewide Trauma Service of Western

Australia, Royal Perth Hospital, WA, Australia

${ }^{3}$ Pharmacology and Anaesthesiology Unit, School of Medicine \& Pharmacology, University

of Western Australia., WA, Australia

${ }^{4}$ Burn Injury Research Unit, School of Surgery, University of Western Australia., WA, Australia

${ }^{5}$ National Drug Research Institute, Curtin University of Technology, Western Australia, WA, Australia

${ }^{6}$ Department of Anaesthesia and Pain Medicine, Royal Perth Hospital, WA, Australia
${ }^{7}$ François-Xavier Bagnoud Center for Health and Human Rights, Harvard School of Public Health, BOSTON, USA

\section{Objectives}

St John's Ambulance together with Royal Perth Hospital and The University of Western Australia investigated how exposure to traumatic scenes at work impacted mental health outcomes among paramedics and pre-hospital workers in rural and remote Western Australia.

\section{Methods}

Questionnaires were distributed evaluating the experience, frequency and nature of dealing with trauma exposure while at work, experience of support services, mood, coping, post traumatic stress, depression, anxiety symptoms, alcohol consumption, general health and well-being, level of work stress and perceived organisational support.

\section{Results}

- Of the most commonly experienced stressful events, pre- hospital workers reported highest levels of fear for unavailability of trained medical personnel, unavailability of suitable transport, and unavailability of reliable communication.

- $55 \%$ and $16 \%$ of respondents reported being exposed to verbal and physical abuse respectively, and $10 \%$ sustained damage to property while on the job.

- $5 \%$ and $3 \%$ of respondents reported post traumatic stress and depressive symptoms above clinical cut-offs on standardised tools.

- $31 \%$ of respondents reported seeing a mental health professional for emotional difficulties.

- $21 \%$ reported taking medication to assist with emotional difficulties

- $11 \%$ were regularly drinking alcohol at harmful levels.

- Job burnout and secondary trauma were both significantly and positively associated with the severity of PTSD and depressive symptoms $(p<0.001)$.

\section{Conclusions}

These early findings suggest that resource limitations unique to rural and remote parts of Western Australia may be among the most stressful traumatic exposures experienced by prehospital workers in these areas. Very few prehospital workers admitted to reported experiencing symptoms suggestive of mental health disturbances on formal measures of psychological function, however nearly a third had sought treatment for emotional difficulties. Further research is required to monitor the effects of exposure to trauma in the pre hospital environment and develop appropriate staff support strategies. 


\section{Recurrent patterns in prehospital adverse events}

Roger Price, Jason Bendall, Jillian Patterson, Paul Middleton

\section{Ambulance Research Institute, Rozelle, NSW,} Australia

\section{Objective}

Adverse events are a major issue for healthcare with 1 in 10 inpatients experiencing an adverse event that results in a prolonged hospital stay, injury or death. Despite being repeatedly identified as a high risk area, little prehospital research exists in this field. This study aimed to examine prehospital adverse events, and determine and describe recurrent patterns.

\section{Methods}

In June 2010, Ambulance Service of NSW paramedics were invited to participate in on online survey. They were asked to recall an adverse event and detail the factors they felt may have contributed to its occurrence. Data were interrogated using regression and principal component analyses (PCA).

\section{Results:}

371 replies were received, $75 \%$ from paramedics with $>3$ years service. This represents a response rate of $10 \%$.

- 8 single components were nominated by paramedics as factors they considered to have contributed to their adverse events, including; a deteriorating patient, decreased level of consciousness (LOC), paramedic uncertainty, and an unusual or understated patient presentation.

- PCA revealed 14 patterns of grouped components but only 2 of these ("Overwhelmed" and "communication") were linked to significant adverse outcomes for patients. The average number of contributory components per/ case was 10 .

\section{Conclusions}

Whilst the applicability of study findings is limited by the low survey response rate, the impact of certain patient characteristics (deteriorating, decreased LOC, unusual or deceptively benign presentation), paramedic features (uncertainty) and situational issues (adapting from low to high acuity or severity) on serious adverse events emerged. The multi-causal and synergistic nature of adverse events and the impact of external factors upon good clinical judgement also became clear. An understanding of this work within the wider human factors literature may help organisations to prioritise their patient safety agenda and build awareness among paramedics about risk prone areas of practice.

\section{MIST AMBO - A minimum data set to improve clinical handover between paramedics and emergency department clinicians \\ Jacinta Young}

\section{Ambulance Service of NSW, Rozelle, NSW, Australia \\ University of Technology, Sydney, NSW, Australia}

\section{Background}

A standardised approach to clinical handover between paramedics and emergency clinicians has recently been developed and implemented across NSW. The new clinical handover tool was initially designed by an Intensive Care Paramedic using the Australian Commission on Safety and Quality in Health Care "OSSIE" Guide to Clinical Handover Improvement. In NSW, paramedics have been using the MIST mnemonic for clinical handover. MIST stands for Mechanism of Injury; Injury or Illness; Signs and Symptoms and Treatment and Times. MIST is an integral part of paramedic handover practice yet evidence has shown it is inadequate for conveying all the necessary information required by emergency clinicians e.g. allergies. To develop a comprehensive minimum data set for clinical handover between paramedics and emergency clinicians that ensures patient safety and continuity of care whilst retaining its uniquely prehospital focus.

\section{Method}

The MIST mnemonic was adapted to become I MIST AMBO. Each letter represents a step in the handover process. The tool can be used for both trauma and medical cases depending on the situation. Identification of the patient; Mechanism of injury or Medical Condition; Injuries or Illness; Signs and Symptoms; Treatment and Trends; Allergies; Medications; Background to event and Other relevant information related to the case.

\section{Results}

The effectiveness of the I MIST AMBO mnemonic was researched by the University of Technology Sydney in collaboration with the Ambulance Service of NSW. A pre and post implementation study of I MIST AMBO was undertaken. Results showed improved outcomes for clinical handover in a number of areas including; information being handed over in a more predictable order; fewer questions being asked by emergency department staff and a reduction in handover duration.

\section{Conclusion}

I MIST AMBO has proven to be an effective mnemonic for clinical handover and is widely accepted by paramedics and emergency clinicians alike. 


\section{Patient Safety in Pre-hospital Emergency Care - A review of the literature Andrea Wyatt}

\section{Ambulance Victoria, Victoria, Australia Monash University, Victoria, Australia}

\section{Objectives}

The quality agenda within health care has a long and evolving history. Influenced by a number of landmark reports the term 'quality assurance' has largely fallen out of favour, and the term 'patient safety' is becoming more accepted. However, to date much of the focus and research concerning patient safety relates to hospitals and the acute inpatient care setting. The pre-hospital environment is characterised by complexity and uncertainty, where the Paramedic is called on to make judgments and decisions that may have significant impact on patient outcomes and patient safety. Therefore it would be expected that there would be a growing interest in quality and patient safety issues in this area. The purpose of this review is to examine the international literature, and determine if ambulance services have made attempts to adopt the 'patient safety' paradigm that is evident across other health disciplines.

\section{Methods}

A literature review was undertaken to explore: What research has been undertaken regarding patient safety in pre-hospital emergency medical care that provides evidence of the adoption of the patient safety paradigm by pre-hospital emergency care services?

\section{Results}

The initial search results identified 548 articles, and following a review of the abstracts and application of the exclusion criteria, 27 articles remained.

\section{Conclusions}

Common themes identified in the pre-hospital patient safety literature include:

- more research needs to be undertaken on patient safety in pre-hospital emergency care;

- the importance of developing a workplace safety culture;

- developing paramedic clinical decision making;

- development of health/pre-hospital data systems;

- development of specific pre-hospital emergency care quality/patient safety indicators.

There is a recognition of many of the key issues identified within other healthcare domains, and at least preliminary initiatives undertaken to address these issues. However more effort still required to align with other health professions and to more rigorously research the highlighted concerns.

\section{Utilisation of extended clinical skills by Paramedic Emergency Care Practitioners at the London Notting Hill Carnival Tim Edwards}

\section{London Ambulance Service NHS Trust, London, UK}

\section{Objectives}

To quantify the use of extended clinical skills by Paramedics with Emergency Care Practitioner ( $P$ ECP) training deployed at the 2007 London Notting Hill Carnival

\section{Methods}

P-ECP staff were equipped with specific P-ECP equipment and deployed at static treatment centres around the carnival route. Each P-ECP was provided with a study specific form to prospectively record brief demographic and treatment details for each patient attended. Service patient report forms were completed contemporaneously and reviewed retrospectively to provide additional clinical information for each case.

\section{Results}

P-ECP staff treated a total of 92 patients over two days. Of these, $73 \%(n=68)$ were treated and discharged, with the remaining $27 \%(n=25)$ referred to hospital. The most common presenting complaints were wounds $(n=32)$, allergic reactions $(n=11)$, soft tissue injuries $(n=10)$ and head injuries $(n=10) .21 \%$ of patients were treated with medications only available to PECPs, including antihistamines $(n=10)$, analgesics $(n=5)$ and antibiotics $(n=4)$. A quarter of patients received treatments or investigations beyond current paramedic scope of practice, with wound closure accounting for the majority of these cases $(n=20)$. Two patients underwent Ear-Nose-Throat and examination and one had urinalysis performed. P-ECPs utilised advanced life support skills in a number of cases, including establishing intravenous infusions $(n=6)$, recording 12 Lead electrocardiograms $(n=6)$ and administering drugs including adrenaline, hydrocortisone and bronchodilators $(n=4)$.

\section{Conclusions}

P-ECPs deployed to static treatment posts are able to teat and discharge an appreciable proportion of patients seeking care at the London Notting Hill Carnival. The management of a quarter of the cases seen required treatments or investigations beyond current UK paramedic scope of practice. A small number of patients were treated using advanced life support procedures, highlighting the need for all practitioners working in a see and treat capacity at events to maintain standard paramedic advanced life support skills. 
Developing a Prioritised Vehicle Equipment Check-sheet (VECS): A modified Delphi Study. Edward Duncan ${ }^{1}$ and David Fitzpatrick ${ }^{2}$

${ }^{1}$ University of Stirling, Stirling UK

${ }^{2}$ Scottish Ambulance Service, Edinburgh UK

\section{Background}

The number, type; and complexity of equipment carried on frontline ambulances is increasing each year. Whilst this enhances the range of prehospital interventions available, it also results in lengthy equipment checks which, on occasion, are interrupted by emergency calls. This can lead to ambulances arriving at an incident without vital equipment, or with equipment that malfunctions. Although equipment check-sheets have previously been developed to support ambulance clinicians, an informal audit of Scottish Ambulance Service practice indicated that these were outdated, unprioritised and not in routine use. To develop a prioritised vehicle equipment check-sheet for routine use

\section{Methods}

Participants: 99 ambulance clinicians were purposively selected and invited to participate.

Design: A modified Delphi study was undertaken. A list of all routine ambulance equipment was collated and developed into the initial Delphi study questionnaire. Participants were then asked to prioritise each item (on a scale from 1 [low priority] to 7 [high priority]) in two rounds. The questionnaire was distributed and returned by email.

Analysis: Means and Standard Deviations were calculated for each item from round two.

\section{Results}

27 participants completed both rounds of data collection. Items which were required for life saving intervention rated the highest. These were followed by items relating to personal protection and infection control.

\section{Conclusions}

This study has enabled the development of a prioritised vehicle equipment check-sheet with high face validity. Its prioritised nature means that vital equipment is accounted for first, ensuring their presence and functionality even if the vehicle is dispatched before a full check can be completed. The check-sheet is now being introduced throughout the Scottish Ambulance Service and would have applicability to other emergency medical services.
An assessment of undergraduate paramedic students' empathy levels: A multi-institutional study

Brett Williams ${ }^{1}$, Malcolm Boyle ${ }^{1}$, Richard Brightwell ${ }^{2}$, Scott Devenish ${ }^{3}$, Peter Hartley, Michael McCall ${ }^{5}$, Paula McMullen ${ }^{5}$, Graham Munro $^{6}$, Peter O'Meara ${ }^{7}$

${ }^{1}$ Monash University, Melbourne, Australia

${ }^{2}$ Edith Cowan University, Perth, Australia

${ }^{3}$ Queensland University of Technology,

Brisbane, Australia

${ }^{4}$ Victoria University, Melbourne, Australia

${ }^{5}$ University of Tasmania, Hobart/Sydney,

Australia

${ }^{6}$ Charles Sturt University, Bathurst, Australia

${ }^{7}$ Latrobe University, Bendigo, Australia

\section{Objectives}

Evidence suggests that improved empathy behaviours among healthcare professionals directly impacts on healthcare outcomes.

However, the 'nebulous' properties of empathic behaviour often means that educators fail to incorporate the explicit teaching and assessment of empathy within the curriculum. This represents a potential mismatch between what is taught by universities and what is actually needed in the healthcare industry. The objective of this study was to assess the extent of empathy in paramedic students across seven Australian universities.

\section{Methods}

A cross-sectional study involving a paper-based questionnaire employing a convenience sample of first, second, third and fourth year undergraduate paramedic students. Student empathy levels were measured using two standardised selfreporting instruments: Jefferson Scale of Physician Empathy (JSPE) (maximum score 140), and Medical Condition Regard Scale (MCRS) (maximum score 66).

\section{Results}

A total of 783 students participated in the study of which $57 \%$ were females. The overall JSPE mean was $106.74(S D=14.8)$. Females had greater mean JSPE empathy scores than males $108.69 \vee 103.58(p=0.042, d=0.37)$. The JSPE also identified that first year undergraduate paramedic mean empathy levels were the lowest, 106.29 with fourth years the highest at 110.60. The lowest MCRS mean score was substance abuse $(M=41.57, S D=12.29)$, and was also statistically significant between genders $(p=0.008$, $d=0.19)$ and universities $(p=0.004, d=0.19)$.

\section{Conclusions}

This study suggests that undergraduate paramedics improve their empathy towards patients over the duration of the course and that they lack compassion for some patients in certain 
situations. These results are significant in the emerging paramedic discipline and provide educators with important data in terms of guiding the paramedic curricula that is directly responsive to requirements of contemporary out-of-hospital healthcare.

\section{Interprofessional graduate program: providing new professional opportunities for nursing - paramedic graduates} Tina Ivanov', Julie Considine ${ }^{1}$, Tony Walker ${ }^{2}$, Fiona Middleton $^{3}$, Danielle Waddell ${ }^{3}$, Vanessa Gorman $^{3}$, Bart Wunderlich ${ }^{1}$

${ }^{1}$ Deakin University-Northern Health Clinical Partnership, Victoria, Australia

${ }^{2}$ Ambulance Victoria, Victoria, Australia

${ }^{3}$ The Northern Hospital, Victoria, Australia

The first double degree graduates with dual qualifications as Registered Nurses and Paramedics completed their studies at the end of 2010 and the numbers of graduates from double degree programs will increase in Australia in coming years. Current nursing and paramedicine graduate programs are discipline specific, defeating the purpose of a double degree and resulting in poor use of graduates' skills and knowledge. In January 2011, a collaboration between Northern Health and Ambulance Victoria resulted in the launch of the Interprofessional Graduate Program enabling graduates to practice as novice practitioners in paramedicine and emergency nursing. This innovative program is an Australian first and presents new opportunities for workforce redesign and graduate experience through interprofessional learning. The program takes advantage of the similarities between emergency nursing and paramedicine but also maintains the unique features of each profession. In addition, the program design ensures alignment between clinical and theoretical content and scope of practice of both disciplines. New health workforce models are vital to sustainability in a rapidly evolving health care system and will enable us to be responsive to the needs of the patients we care for. The Interprofessional Graduate Program challenges traditional notions of graduate programs in both nursing and paramedicine and provides alternative experience for graduates who are going to be future senior clinicians. In this paper, the development and implementation of the Interprofessional Graduate Program will be outlined and the preliminary outcomes from the first six months of the program will be presented.

\section{The Professional Socialisation of Paramedics: The Transition from University Student to Paramedic Intern.} Scott Devenish ${ }^{1}$, Michele Clark ${ }^{2}$, MaryLou Fleming $^{3}$, Stephen Loftus ${ }^{4}$

${ }^{1}$ Lecturer, Paramedic Practice, School of Public Health, Queensland University of Technology, Brisbane, QLD, Australia

${ }^{2}$ Director of Research, School of Public Health, Queensland University of Technology, Brisbane, QLD, Australia ${ }^{3}$ Head of School, School of Public Health, Queensland University of Technology, Brisbane, QLD, Australia ${ }^{4}$ Deputy Director, Education for Practice Institute, Charles Sturt University, Sydney, NSW, Australia

\section{Objectives}

Professional socialisation is defined as the process by which professionals learn the values, behaviours and attitudes necessary to assume their chosen professional role. The socialisation process consists of three distinct phases namely, pre-socialisation (Anticipatory), socialisation (Encounter) and post-socialisation (Change and Acquisition) phases. This research explores the lived experience of paramedic interns, during their encounter phase of their professional socialisation, as they make the transition from university student to paramedic intern.

\section{Methods}

Participants were sought from several of Australia's larger ambulance services and UK NHS Ambulance Trusts to take part in this study. Participants were recruited through Ambulance Service Research Institutes, Clinical Governance Departments and university databases. To be included in this study, participants were required to be university educated, be employed in a professional internship year and to not have achieved qualified/registered paramedic status. Data collection was via face-to-face semistructured interviews. A thematic analysis of the interview transcripts using narrative inquiry has been employed.

\section{Findings}

The excitement of gaining employment with an ambulance service was equalled by fear. Once employed, paramedic interns encountered the 'reality shock' of full-time work. They were confronted with the ambulance culture, and felt the need to conform to this culture in order to be accepted by station colleagues and management. Paramedic interns learned to cope with a range of challenging situations, and to deal with the reality of ambulance practice where not every case was an urgent life saving endeavour. 


\section{Conclusions}

In the encounter phase of professional socialisation, the transition from university student to paramedic intern can be a very difficult experience. Interns felt the need to assimilate into the culture to be accepted, and there was a perceived dissonance between the university and ambulance cultures.

\section{What is the undergraduate paramedic students' perspective on the acceptability, realism, and effectiveness of Simulated Patients? \\ Matt Johnson, Leanne Boyd, Jade Sheen}

\section{Monash University, Victoria, Australia}

\section{Background}

This paper reports on a pilot study, exploring students' views regarding the strengths and weaknesses of Simulated Patients (SPs) in an undergraduate paramedic degree. Research into the theory practice gap of paramedic students has found less than 50 per cent of cases experienced during clinical placement lead to skill practice on a real patient. The goal of any clinical experience, be it in a placement or a simulation, is to provide a context within which clinical concepts can be brought together and practiced. Without genuine student engagement - that is, with the students acting only as observers - clinical placements may offer little more than face validity. This represents a model of competence training that is increasingly being seen as unsustainable. An alternative that may offer content validity is to create a controlled situation that nonetheless offers an immersive experience where participants are able to suspend disbelief leading them to speak and act as if the situation were real. The potential of simulations to enhance learning has been recognised at a Federal level with Health Workforce Australia (HWA) undertaking a \$94M Simulated Learning Environments (SLE) program.

\section{Method}

A cross-sectional survey of second year paramedic students. This study sought to explore the perceptions and reactions of paramedic students to a Simulated Patients and views the data through a qualitative lens.

\section{Results}

Students indicated that the scenarios with Simulated Patients afforded a greater sense of reality than the use of peers and the results raise the potential for high fidelity scenarios using SPs to create a controlled, environment that may allow for learning goals previously attributed to clinical placements to be met within the academic curriculum.

\section{Conclusion}

The question of whether intended clinical placement learning outcomes can be feasibly met by increasingly the fidelity of simulations using SPs warrants further exploration.

\section{Educating Gibraltar: The challenges of bringing university education to Scott Stewart}

Victoria University, Melbourne, Australia St Georges University of London, London, UK

\section{Objectives}

To provide a tiny, remote ambulance service with world standard education.

\section{Methods}

An overview of the challenges faced by the Gibraltarian Ambulance Service and innovative methods used to facilitate their university participation.

\section{Results}

The Gibraltar service has limited scope of practice and education. Its island like isolation, due to political forces, means it faces challenges akin to a remote small town. It has additional risks in having large influxes of tourist via cruise ship, an international airport near town and a busy shipping lane. Historically only BLS was considered necessary due to the short transport times. The skill / education gap has now been recognised. However traditional university attendance was not possible due to staffing and geographic constraints. Tertiary education has been commenced by burst, mixed mode delivery. This mixes visits by UK staff with an online learning environment combining traditional text based resources and links with 'Wimba' live class room and interactive 'Second Life' scenarios. Assessment is a mix of face to face OSCE, essays and online tests. The first year of delivery resulted in high student satisfaction and academic performance. Changes in practice and professional interaction have been noted.

\section{Conclusions}

Gibraltar provides a fascination microcosm of paramedic work. Hurdles have been overcome to enable tertiary level education for this remote British Overseas Territory. Positive changes are happening. 
The UK paramedic Education system:

Learning's of an Australian paramedic academics experiences teaching at St Georges University, London

Scott Stewart

Victoria University, Melbourne, Australia

St Georges, University of London, London, UK

\section{Objectives}

To gain an understanding of the differences between the Australian and UK paramedic systems with a focus on education.

\section{Methods}

An overview of the notable differences and learning's gained from teaching at St Georges University London, and working with UK Paramedics for two years from the perspective of an Australian Paramedic Academic is presented.

\section{Results}

Once the variances in jargon are stripped away, the most striking finding is the similarity of AngloAmerican style Ambulance Services world wide. Patients, staff and problems faced are remarkably similar. The UK paramedic system has differences in registration, education, guidelines, drugs used, Paramedic practitioners and the frequent presence of medical doctors in the out of hospital environment. It also has recently adopted a Victorian style trauma system and intensive care paramedics. My personal insight is that the Australian system is world class. Australian Paramedics and their universities are well regarded internationally. The transition to teaching in the UK is significant but not prohibitively difficult. The art of teaching in different system is akin to learning a new language; the structure of the process becomes clear. For Australian paramedics wishing to work in the UK the task is well mapped but long and moderately expensive. For paramedic Academics the transition is slightly easier.

\section{Conclusions}

The UK system is accessible and worthwhile for Australian paramedics and paramedic academics. The experience suggests an ethnological study of paramedics and lecturing staff that have made the transition from one region to another. 


\section{Conference Poster Abstracts}

\section{Logging onto a clinical focused course. Liz Thyer and Georgia Clarkson}

Victoria University, St Albans, Victoria, Australia

\section{Objectives}

Our study aims to catalogue paramedic student expectations of distance education prior to undertaking such a course and then again after completion of a year on distance education. We will also gather information regarding the alignment of these expectations with reality as well as production of distance education material and resources, student resources, age, gender and location of students. Furthermore this research aims to identify if there is any impact on student grades by distance education.

\section{Methods}

The project employed a mix methodology quasiexperimental design encompassing two questionnaires and collation of student grades (grade point averages - GPA's) pre and post distance education experience. A pilot study was conducted during the first year of the study and a larger study is currently being undertaken. Participants were third year students enrolled in the Bachelor of Health Science (Paramedic) at Victoria University completing components of their study on-line.

The two questionnaires utilised a Likert scale with additional directed open-ended questions.

Categorical data was analysed using frequency distribution tables and determination of the mode for each question whilst data collected from openended questions was subject to basic ethnographic analysis.

Human ethics approval was obtained from Victoria University (HRETH 10/6).

\section{Results}

Responses to the pilot study indicated student felt online learning was appropriate for some theoretical components of paramedic practice; however many students identified issues with their time management, technological barriers and decreased engagement. Positive responses were elicited from students in relation to flexibility of learning and ability to re-visit information as well as environmental savings.

\section{Conclusions}

Initial anxieties regarding online learning were not realised and participants in the study largely expressed positive overall online learning experiences where negatives were outweighed by positive feedback.
The study identified a number of student concerns that could be used to inform improved future teaching practice.

\section{The efficacy of a double degree program regarding student satisfaction: perspectives of undergraduate students. Yelise Foon and Natasha Nemarich}

\section{Charles Sturt University, Bathurst, Australia}

\section{Introduction}

Selected tertiary institutions offer the unique availability to study pre-hospital care and nursing as a combined program. Currently, Australia-wide, four universities offer a Bachelor of Nursing in combination with a Bachelor of Clinical Practice (or equivalent) - Charles Sturt University, Queensland University of Technology, Australian Catholic University and Monash University. Within Charles Sturt University the double degree is offered over four years as an internal student. From course commencement to completion, students undergo dynamic shifts within their learning experiences, which may effect their perceived sense of worth when undertaking dual qualification study.

\section{Hypothesis}

Current students have varying levels of satisfaction depending on mitigating factors influencing the course of their study in the double degree program.

Objective: To assess the efficacy of the Bachelor of Nursing/Bachelor of Clinical Practice (Paramedic) combined degrees at Charles Sturt University (CSU) when considering the satisfaction of current students and perceptions of differing grade subsets.

\section{Methods}

A survey of current double degree students enrolled in their first to fourth year of study will be conducted. The questions are specifically designed to review individual motivation for pursuing dual qualifications and factors within the double degree program impacting student satisfaction. These questions will be quantitative, displayed as graphical trends, as well as qualitative, collated in themed data sets and independently reviewed by a team of two individuals. The sample size of the survey is 100 students $(n=100)$ across four different years.

\section{Conclusion}

The projected timeline of this study will include the open survey from July $1^{\text {st }}$ - July $31^{\text {st }}, 2011$. From August $1^{\text {st }}-15^{\text {th }}$, the data will be collated and analysed, and a completion date of $31^{\text {st }}$ of August. Concurrently, an independent party will review the data for bias. 
Pain assessment and pain management practices of ambulance officers in Auckland.

Sarah Werner ${ }^{1}$ and Jane Koziol-McLain ${ }^{2}$

${ }^{1}$ St John, Auckland, New Zealand

${ }^{2}$ AUT University, Auckland, New Zealand

\section{Background}

Pain is a common complaint of many patients in the prehospital setting. Pain prevalence is unknown in Auckland, New Zealand. Little is known about how ambulance officers assess and treat pain. Ambulance officers have more options than previously to treat pain, but actual practice is unknown.

\section{Objectives}

This study sought to establish the prevalence of patients reporting pain, characteristics of pain assessment, including pain scores and OPQRST, and treatment of pain using pharmacological and non-pharmacological interventions. Relationship between qualification and pain assessment practices was sought. Data gathered will provide a baseline for future research.

\section{Methods}

Patient report forms were randomly selected over a 7-day period $(n=371)$ in September 2010. Data abstracted included presence of pain, pain assessments documented including OPQRST and pain scores, pharmacological and nonpharmacologic treatment of pain documented. Descriptive statistics were used to analyse the data. Deceased patients, patient transfer patients, and accidental personal alarm activation cases were excluded from analysis.

\section{Results}

Pain was reported in $49 \%$ of eligible patients; the most common location being chest pain. Initial pain scores were documented in $20 \%$ of patients in pain. Time $(50 \%)$ followed by Onset $(39 \%)$ were most frequently reported pain assessments. Low rates of analgesia administration were reported for those reporting pain (paracetamol 19\%, Entonox $^{\text {TM }} 16 \%$ ). Intravenous morphine was administered to $8 \%$ of patients reporting pain, mean dose $7.5 \mathrm{mg} \pm 5.7$. Analgesia refusal was noted in $6 \%$ of patients. Non-pharmacological treatment of pain was very poorly documented, positioning (4\%), followed by splinting (3\%). Conclusions: Assessment of pain is poorly documented. Low rates of analgesia administration are present. Non-pharmacological treatment of pain appears undervalued. Further research opportunities exist about the patient's desire for analgesia, and pain assessment in paediatric and cognitively impaired patients.

\section{Tabletop Exercises for Paramedic Incident} Command Training

Christopher R. Foerster

James Cook University, Townsville, QLD, Australia

Lambton EMS, Lambton County, Ontario, Canada

\section{Background}

Paramedics and supervisors have an important command role in coordinating the medical response to multiple casualty incidents (MCls). Tabletop exercises are an effective and economical disaster exercise format that are commonly used to prepare upper level emergency management personnel for their decision-making role in disasters. An adapted version of this exercise style may be useful for preparing paramedics for their front-line command role in MCls.

\section{Objectives}

The objective of this project was to investigate the feasibility of developing and delivering a tabletop exercise for paramedics.

\section{Methods}

A tabletop exercise for paramedics was developed around a scenario of a suicide bombing at a sports stadium during an event. The response was divided into three modules with the exercise taking place over two hours including the introduction and debriefing. The author facilitated two pilot tests of the program with different ambulance services, one with front-line paramedics participating and one with paramedic managers and supervisors.

\section{Results}

The tabletop exercise format was readily adapted to the needs of paramedics. After the initial exercise development, delivery required only limited resources. Exercise materials were minimal, allowing for the exercise to be as mobile as the facilitator. Participants rated the exercise positively, saying that they found it useful to be able to discuss these decisions in a low stress environment. The primary limitation of this study is that it sought to examine only the feasibility of this type of training for paramedics and not its efficacy.

\section{Conclusions}

The results from these pilot tests suggest that tabletop exercises can be adapted to train paramedics for their front-line command role and that delivery requires only minimal resources. Having demonstrated the feasibility, further research is warranted to evaluate the efficacy of this type of training for paramedics. 


\section{Quality of Life of Young Adult Out-of Hospital Cardiac Arrest Survivors Conor Deasy, Janet Bray, Karen Smith, Stephen Bernard, Peter Cameron}

DEPM, Monash University

Alfred Hospital, Melbourne, Australia

Ambulance Victoria, Melbourne, Australia

\section{Background}

Amongst patients suffering out of-hospital cardiac arrest (OHCA), young adults represent a minority. However, these victims suffer the catastrophe when they are in a very active phase of life and have a long life expectancy. Little is known on the quality of life of these survivors. There is no consensus about which particular dimensions should be included in quality of life studies, as well as for the instruments that should be used. Victorian Ambulance Cardiac Arrest Registry has commenced collecting quality of life data using SF-12, EQ-5D and GOSE on OHCAs.

\section{Aims}

To assess the Quality of Life in young adults who survive OHCA in Victoria.

\section{Methodology \\ The Victorian Ambulance Cardiac Arrest Registry (VACAR) records will be used to identify cases of OHCA that occurred between 2003 AND 2008 in the 16-39 year old age group. \\ Survivors will receive a telephone questionnaire to establish their quality of life post cardiac arrest using SF-12, EQ-5D and GOSE.}

\section{Results}

We will present characteristics of young adult OHCAs. We will describe the quality of life outcomes and relate them to the characteristics of the cardiac arrest. We will describe the quality of life tools, their performance, training, policy and procedure around telephone follow up as pertains to this patient group as well as the challenges encountered.

\section{Bullying and Harassment in Paramedicine - One Person's Journey Allison Ballard}

University of Canberra, Canberra, Australia Australian National University, Canberra, Australia

University of South Australia, Adelaide, Australia

This paper will outline a case study/studies in bullying and harassment within different Australian ambulance services and will address areas such as the adverse impacts on career, health and wellbeing, peers, service provision, the "profession", and health organisations. It will also address some of the possible reasons that organisations and individuals fail to really "get" bullying and harassment when it happens to others (or when they are perpetrators), and yet, can easily recognise it when it happens to them or to those close to them.

\section{Prehospital acupressure by paramedics for management of acute pain: a systematic review of randomised controlled trials.}

Paul Simpson, Ric Thomas, Jason Bendall

Ambulance Research Institute, Ambulance Service of New South Wales, Sydney, Australia

\section{Objective}

Pharmacological analgesia is not always available in the prehospital setting, leading to consideration of non-pharmacological alternatives. Acupressure has been studied in several European ambulance services and may be an effective intervention when performed by paramedics. The objective of this study was to conduct a systematic review of the literature to determine the effectiveness of acupressure for acute pain when performed by paramedics in the prehospital setting.

\section{Methods}

A systematic search of MEDLINE, EMBASE, CINAHL and the Cochrane Library databases was conducted to identify prehospital randomised controlled trials comparing paramedic-performed acupressure to 'sham' acupressure for management of acute pain. Potentially relevant articles were systematically assessed for eligibility against a priori inclusion criteria, and for quality and validity using recognised methods as recommended by the Cochrane Collaboration.

\section{Results}

The search identified three small prospective randomised controlled trials that met the inclusion criteria, with a total of 62 patients receiving acupressure and 56 receiving 'sham' treatments. All three compared paramedic-performed 'real' acupressure with 'sham' acupressure using a visual analog scale (VAS) to measure pain reduction and all were methodologically robust in design. One study used auricular acupressure for acute hip fractures in older patients, one used acupressure at the Baihui/Hegu points in patients with radial fractures, while the third used auricular acupressure for fractures and soft tissue injuries. All three studies reported statistically significant differences in post-intervention VAS pain scores favouring 'real' acupressure. Insufficient data was available in the published papers to allow metaanalysis. 


\section{Conclusion}

Paramedic-performed acupressure is an effective non-pharmacological intervention that provides clinically meaningful reductions in acute pain. Acupressure could be a useful addition to the scope of practice of paramedics, particularly those unable to administer analgesics, and nonambulance first aid providers.

\section{Prehospital acupressure by paramedics for managing acute anxiety: Systematic review and meta-analysis of randomised controlled trials.}

Paul Simpson, Ric Thomas, Jason Bendall

Ambulance Research Institute, Ambulance Service of New South Wales, Sydney, NSW, Australia

\section{Objective}

Paramedics commonly encounter patients experiencing acute anxiety. Anxiety secondary to acute pain is often indirectly managed through administration of opioids or other analgesic agents; however, for acute anxiety of non-pain origin, or painful conditions when pharmacological analgesia is not available, paramedics have few anxiolytic avenues other than simple reassurance. Acupressure has been proposed in several European studies to be a simple, effective and safe option that could easily be added to paramedics' scope of practice. The aim of this systematic review was to determine the effectiveness of acupressure for reducing acute anxiety when performed by paramedics in the prehospital setting.

\section{Methods}

A systematic literature search of MEDLINE, EMBASE, CINAHL and the Cochrane Library was conducted to identify prehospital randomised controlled trials comparing paramedic-performed acupressure to sham acupressure for management of anxiety. Identified studies were assessed for eligibility against a priori inclusion criteria, and for quality and validity using recognised methods. A fixed-effect model metaanalysis of methodologically homogenous studies was conducted using Revman 5.1 to provide a summary statistic for mean difference in final (post-treatment) $100 \mathrm{~mm}$ visual analogue scale (VAS) value.

\section{Results}

The search identified five eligible studies comparing 'real' acupressure with 'sham' acupressure, all of which were methodologically robust in design. All studies reported significantly lower post-treatment anxiety scores in the 'real' acupressure groups. Four studies reported sufficient detail allowing extraction of data for inclusion in the meta-analysis. The meta-analysis of 206 patients showed that 'real' acupressure produced a final VAS score about $30 \mathrm{~mm}$ lower than 'sham' acupressure $(31 \mathrm{~mm} ; 95 \% \mathrm{Cl} 25 \mathrm{~mm}$ to $37 \mathrm{~mm} ; \mathrm{p}<0.0001$ ).

\section{Conclusion}

Paramedic-performed acupressure is an effective non-pharmacological intervention that reduces acute anxiety. Acupressure could be easily implemented into paramedics' scope of practice.

\section{Prophylactic metoclopramide for patients receiving intravenous morphine in the emergency setting: A systematic review and meta-analysis of randomised controlled trials. Paul Simpson, Jason Bendall, Paul Middleton}

Ambulance Research Institute, Ambulance Service of New South Wales, Sydney, NSW, Australia

\section{Objective}

The objective of this study was to conduct a systematic review and meta-analysis of randomised controlled trials, comparing metoclopramide to placebo, for preventing vomiting in patients who have received intravenous morphine for acute pain in the emergency setting, and to determine the level of evidence supporting the use of prophylactic metoclopramide in this population.

\section{Methods}

Comprehensive systematic electronic searches were conducted of MEDLINE, EMBASE, and the Cochrane Library for randomised controlled trials addressing the clinical question. Reference lists of identified articles were hand-searched. Studies meeting the inclusion criteria were assessed for validity and bias using Cochrane methodology. Methodologically appropriate clinical trials were included in the meta-analysis using Revman 5.2 software to provide a pooled estimate of effect expressed as odds ratio (OR) with $95 \%$ confidence interval $(\mathrm{Cl})$.

\section{Results}

Three randomised controlled trials fulfilled the search criteria. All three studies were included in the final meta-analysis totalling 595 patients. The incidence of vomiting was $2 \%$ and $3 \%$ for metoclopramide and placebo respectively. The meta-analysis demonstrated an overall result of no difference between metoclopramide and placebo for the primary outcome of vomiting (OR $0.72 ; 95 \% \mathrm{Cl} 0.11-4.58 ; p=0.73$ ) with no evidence of heterogeneity $(p=0.13)$. 


\section{Conclusion}

The incidence of vomiting following administration of intravenous morphine is very low. There was little evidence that routine prophylactic administration of metoclopramide following the administration of intravenous morphine for acute pain management in the emergency setting is clinically beneficial. Routine metoclopramide administration may expose patients to a risk of harm which is not justifiable given a lack of evidence of benefit.

\section{The 'CATCH' study: A preliminary analysis describing the epidemiology of $\mathbf{5 0 0}$ prospectively collected cases involving older fallers attended to by paramedics.} Paul Simpson ${ }^{1}$, Jason Bendall, Jillian Patterson $^{1,2}$, Anne Tiedemann ${ }^{3,5}$, Paul Middleton $^{1,6}$, Jacqueline Close ${ }^{3,4}$

${ }^{1}$ Ambulance Research Institute, Ambulance Service of New South Wales, Sydney, NSW, Australia

${ }^{2}$ New South Wales Department of Health, New South Wales, Australia

${ }^{3}$ Neuroscience Research Australia, Randwick, New South Wales, Australia

${ }^{4}$ Prince of Wales Clinical School, University of New South Wales, Sydney, New South Wales, Australia

${ }^{5}$ The George Institute for Global Health, Sydney, New South Wales, Australia, ${ }^{6}$ University of Sydney, Sydney, New South Wales, Australia

\section{Objective}

Older patients who have fall constitute a significant proportion of ambulance emergency responses. The 'CATCH' study is a large, ongoing prospective descriptive study that aims to describe the epidemiology of 1500 older fallers aged 65 years or more who were attended by paramedics in New South Wales, Australia. The aim of this preliminary analysis is to describe the epidemiology of the first 500 cases enrolled in the CATCH study.

\footnotetext{
Methods

We selected the first 500 cases from the prospective CATCH study database for a preliminary analysis. A tailored data collection tool has been completed for all eligible older fallers, providing falls-specific data not routinely captured in ambulance clinical records. This data was linked ambulance clinical records and computer aided dispatch (CAD) data. Analysis was conducted using SAS 9.2 to produce descriptive statistics and compare proportions.
}

\section{Results}

For the 500 cases included, $76 \%$ were classified as 'falls' by the CAD system, with $53 \%$ receiving an urgent response. The mean age was $83( \pm 7)$, and $64 \%$ were female. Falls happened at home in $54 \%$ of cases, with $18 \%$ occurring in residential care. A simple trip (24\%) and loss of balance $(32 \%)$ were the most common reasons for falling. Almost $30 \%$ of patients spent more than 30 minutes on the floor prior to ambulance arrival, and $78 \%$ sustained a new injury. Almost half had never called for falls previously, while $15 \%$ had called within the last 4 weeks. The overall nontransport rate was $26 \%$ with no statistically significant difference between day and night $(24 \%$ v $32 \%$; $=0.07$ ).

\section{Conclusion}

Ambulance responses to older fallers generally involve females living at home, and one in four results in non-transport. Future research should focus on safe management of non-transported fallers and strategies to optimise the dispatch process to ensure an appropriate response priority.

\section{Proposed studies of Prehospital Sepsis Care} Richard Thomas

Ambulance Research Institute, Rozelle, NSW, Australia

\section{Background}

Sepsis is responsible for the deaths of thousands of Australians annually and leaves many others dependent on high-level hospital care for long periods. The human and financial cost is significant yet reducible. Sepsis describes a number of conditions that originate in an infective source, progressing to organ and systemic cardiovascular failure. Little is described about prehospital recognition and care of community acquired sepsis. Paramedic capacity to identify sepsis has not been described in the literature though in-hospital recognition is known to be difficult. A suite of in-hospital interventions are described that reduce mortality and morbidity, and time-to-care is a critical feature in the management of sepsis. Researchers have begun to examine the potential role of prehospital care to achieve similar mortality and morbidity reductions as have been observed with the contemporary prehospital care of AMI, stroke, and trauma patients.

\section{Methods}

A series of studies are proposed to bridge the knowledge gap and explore potential morbidity and mortality reductions through prehospital intervention. The first study, already underway, is an epidemiological review of close to 3 million cases in NSW to describe the prehospital 
incidence of sepsis including frequency, distribution, and mortality. The second will examine the capacity of paramedics to identify septic patients and validate an assessment tool. A third study is a randomized trial that will compare standard care versus high-volume fluid administration. A fourth and final study will look at prehospital antibiotics for sepsis.

\section{Results}

These proposed studies will advise who is septic, when and where; if paramedics can identify them; and if increased prehospital fluid volumes and antibiotics will reduce mortality and morbidity.

\section{Conclusions}

Studies that improve the understanding of sepsis and identify interventions that can begin at the earliest time, during the prehospital period, may lead to reductions in mortality, morbidity and health care costs.

\section{Paramedic Decision Making: Issues in Rural Settings Kate Kloot Cleverley}

Ambulance Victoria, Warrnambool, Australia Deakin University, Centre for Rural Emergency Medicine, Warrnambool, Australia

\section{Background}

Clinical decision-making plays an important role in the pre-hospital healthcare environment. It allows paramedics to gather and process information, problem-solve and choose a treatment course for their patient. Despite guideline and protocol use, patient management still requires some form of judgement regarding the patient's condition. Whilst significant research has been undertaken into nursing and general medical decision-making processes, very little has examined the problemsolving methods utilised by paramedics.

\section{Discussion}

Decision making efficacy is dependent on clinician experience level and the presence of stressors (e.g. time pressure or noisy environments), as they change cognition demands. Increased experience allows clinicians to use heuristics (cognitive shortcuts) and better identify relevant cues in busy or stressful environments, but stressors are often increased in rural areas due to distance and resource limitations. The seriously injured or unwell patient often has a combination of complex medical problems, a greater risk of death, and is more likely to rapidly deteriorate during transport due to longer transport times. Not only are there increased cognitive demands based on the patient's condition, but issues such as patient history location, resource availability, paramedic skill set, and patient/family transfer requests are all factors needing consideration.

\section{Conclusions}

The paramedic's ability to gather information, problem-solve and select a course of management are of particular importance in timeand resource-limited settings; two situations common in the rural pre-hospital environment. The paramedic's experience level and the presence of stressors will affect cognitive demands, in turn affecting the decisions made and patient outcome. This is more significant in rural areas due to decreased resource availability and increased travel time to appropriate care. Future research needs to be undertaken in the pre-hospital setting to ensure optimal paramedic clinical decision making processes are identified and taught in order to provide best patient care.

\section{Alternate Care Facility Triage}

Christopher R. Foerster ${ }^{1,2}$, Adam Perper ${ }^{3}$, Bethany Cummings ${ }^{4}$, Steven J. Parrillo ${ }^{5}$, Erik S. Glassman $^{6}$

${ }^{1}$ Lambton EMS, Lambton County, Ontario, Canada

2James Cook University, Townsville, QLD, Australia

${ }^{3}$ Hackensack University Medical Center, Hackensack, NJ, U.S.A.

${ }^{4}$ Department of Emergency Medicine, Winchester Medical Center, Winchester, VA, U.S.A.

${ }^{5}$ Department of Emergency Medicine, Einstein Hospital Elkins Park, Philadelphia, PA, U.S.A. ${ }^{6}$ Strasburg Rescue Squad, Strasburg, VA, USA

\section{Background}

Despite implementation of surge capacity plans, mass casualty incidents will tax the resources of surrounding healthcare facilities. Lower acuity patients can deplete resources both on scene and in emergency departments. Current prehospital triage schemes prioritise patients for transport, but do not identify patients who can be safely transported to Alternate Care Facilities (ACFs) to ease the burden on the traditional points of entry.

\section{Objectives}

The objectives of this project were to identify patient populations that can be safely transported directly to ACFs and to develop an algorithm for use by field providers making this determination.

\section{Methods}

Past mass casualty incidents were considered to determine which patient populations could be transported directly to ACFs. Patient populations were sought that required minimal care but may needlessly utilise resources at a traditional point of entry and on scene. An algorithm was then developed for use by field providers to determine patients' suitability for transport to an ACF. 


\section{Results}

Four primary patient populations were identified who could be eligible for transport to alternate care facilities. After activation by the Incident Commander, patients who are classified by the traditional triage scheme as walking wounded, worried well, or requiring delayed urgent care would be considered for inclusion. Specific groups who may be eligible are those with isolated soft tissue injuries, isolated orthopedic injuries, isolated mental health issues, or multiple minor injuries or illness. Precise implementation of this algorithm is dependent on local resources, legislation and regulations. This initial work now requires validation in a full-scale exercise.

\section{Conclusions}

Transport to ACFs can direct a specific patient population away from traditional points of entry to enhance existing surge capacity plans. The algorithm presented in this research can be used as a basis for local protocols for field providers to determine which patients should be transported to ACFs.

\section{Curricula Coherence: Is Constructive Alignment a potential solution in paramedic education? Brian Sengstock ${ }^{1}$ and Brett Williams ${ }^{2}$}

${ }^{1}$ University of the Sunshine Coast, Queensland, Australia

${ }^{2}$ Monash University, Melbourne, Australia

Universities around the world are experiencing significant challenges as they position themselves in competitive knowledge-based economies. On a national scale, these challenges, possible solutions, and potential roadmaps have been outlined in the Building University Diversity and the Bradley Reports which examined the approval, registration, and flexibility issues relating to the Australian Higher Education sector. These challenges are having a major impact on those involved in paramedic education and training. As the number of Australian paramedic programs continues to grow, it is timely that academics, policymakers and educators consider the implications of not having national curricula standards or agreed upon graduate attributes.

Currently, no national curricula conformity, standardisation, or graduate attributes exists, which suggests that Higher Education institutions and industry are uncertain if graduates are meeting their respective needs. Thus, an opportunity for curricula renewal, informed by curriculum design theory is now available for those involved in paramedic education and training. One such theory is Constructive Alignment. Constructive alignment is a curriculum design theory that aligns graduate attributes, intended and observed learning outcomes, teaching and learning activities and standardsbased assessment.

Constructive alignment appears to offer curriculum designers and professional accreditation bodies the necessary theoretical framework to ensure the consistent implementation of graduate attributes. Evidence indicates that simply adding graduate attributes or psychomotor skills on an ad-hoc basis or as a bolt-on to existing courses does not provide the contextualised learning processes required. Additionally, undertaking course mapping exercises has been likened to a 'tick and flick' approach and in isolation is ineffective in ensuring that espoused graduate attributes are experienced and demonstrated by students. This paper will provide examples of how constructive alignment has been used by cognate and non-cognate disciplines, providing the paramedic discipline with a roadmap in its urgent need of curricula coherence.

\section{Transition to tertiary education: Preliminary findings from first year paramedic students Brett Williams and Fiona Newton}

Monash University, Melbourne, Australia

\section{Objectives}

As part of its 20/40 agenda, the Australian Commonwealth government has proposed that $40 \%$ of 25 - 34 year olds will complete a Bachelor's level qualification by 2020 . This target is now placing greater emphasis on transitional issues to tertiary education. While a number of studies have established the importance of positive transition experiences as a foundation for retention, to our knowledge, no such studies have examined this aspect from a paramedic perspective. The aim of this study was to survey paramedic students as they make their transition into first year tertiary studies at a large Australian University.

\section{Method}

A cross-sectional study consisting of a paperbased questionnaire (115 items) using a convenience sample of first year undergraduate paramedic students. Student self-directedness was assessed using a unipolar 5-point Likert scale (1=Strongly Agree to 5=Strongly Disagree), while confidence in a number of information technology and assignment preparation skills were measured using a 7-point bipolar Likert scale (ranging from ${ }^{-}$ 3 to $\left.{ }^{+} 3\right)$. 


\section{Results}

Twenty-four students participated in the study (40\% response rate). The median age was 23 years, the majority of students were female $n=17$ (70\%). The median ATAR score was 90.0 . Students responded positively to selfdirectedness, for example, 'I want to learn new information' ( $\mathrm{M}=1.25, \mathrm{SD}=0.44)$, and 'I am responsible for my own decisions' ( $M=1.45$, $\mathrm{SD}=0.58$ ). Students lacked confidence in being able to find resources from library databases: 'Ability to narrow search using Boolean terms' $(\mathrm{M}=0.87, \mathrm{SD}=1.82)$, 'Ability to broaden search using truncations' (M=0.52, $S D=2.04)$, and 'Download a full text article from a database' $(\mathrm{M}=0.47, \mathrm{SD}=2.06)$.

\section{Conclusion}

This information will provide teaching staff with important data that will be used to facilitate the design of future transition programs. It is anticipated that the data will also facilitate staff in making improvements in teaching and learning within the paramedic program.

\section{Oxygen therapy in Acute Coronary Syndromes: Are we doing more harm than good?} Jennifer Melvin ${ }^{1}$, Ziad Nehme ${ }^{2}$, Brett Williams ${ }^{2}$

${ }^{1}$ The University of Notre Dame, Perth, Australia

${ }^{2}$ Monash University, Melbourne, Australia

\section{Objectives}

Administration of oxygen therapy for patients suffering acute coronary syndrome (ACS) has for many years taken a leading role in paramedic clinical practice guidelines. However, this practice is based on a false premise of benefit rather than reflecting evidence-based practice. We conducted a review of the literature to determine whether normobaric oxygen therapy is an effective treatment for ACS.

\section{Methods}

A literature review was undertaken using the electronic databases PubMed, Ovid Medline, CINAHL Plus and the Cochrane Library from their commencement to the end of May 2011. Keywords used in the search included; acute coronary syndrome, myocardial infarction, heart attack, MI, AMI, heart infarct, angina, coronary syndrome, coronary thrombosis, and oxygen. Clinical trials only were eligible for inclusion if they measured the effect of normobaric oxygen therapy on patients suffering ACS.

\section{Findings}

Of the initial 4765 articles, 66 potential studies were identified after screening title and abstract. From these, 2 clinical trials met the eligibility criteria. The use of high flow oxygen therapy in uncomplicated myocardial infarction was associated with a non-significant increased risk of mortality (risk ratio $2.0,95 \% \mathrm{Cl} 0.8$ to 10.3 , $p=0.08$ ), and a greater aminotransferase level $(p=0.05)$ when compared with room air. When guided by pulse oximetry, severe hypoxaemia $\left(\mathrm{SpO}_{2}<80 \%\right)$ was less frequent in myocardial infarction patients treated with oxygen ( $4 \%$ vs. $35 \%, p<0.05)$.

\section{Conclusion}

There is no empirical evidence to support the routine use of oxygen therapy for ACS. Limited evidence suggests that oxygen therapy limits hypoxaemia within 24 hours of onset of myocardial infarction; however it may also lead to an increase in mortality.

\section{The emergency ambulance workload during the early recovery periods from the Christchurch earthquakes. Bronwyn Tunnage ${ }^{1}$ and Mark Deoki ${ }^{2}$ \\ ${ }^{1}$ AUT University, Auckland, New Zealand \\ ${ }^{2}$ St John New Zealand, Auckland, New Zealand}

\section{Objectives}

An earthquake measuring 7.1 in magnitude struck the city of Christchurch on 4 September 2010 causing widespread damage. Aftershocks continued for months followed by a 6.3 magnitude earthquake on 22 February 2011 which resulted in multiple fatalities and major damage. At a time when businesses and organisations had great difficulty maintaining their usual activity, continuity of provision of pre-hospital emergency health care was critical. Many of the local population were bereaved or recovering from injury as well as living with considerable social and economic disruption. The literature reports that increased incidence of cardiac and psychological disorders have been observed for months after earthquakes.

This study quantifies the emergency ambulance workload and performance in the months following the Canterbury earthquakes. It establishes the change in demand for ambulance responses overall, and by priority response categories, and the change in response time key performance indicators $(\mathrm{KPI})$, compared to previous years.

\section{Methods}

A retrospective analysis was undertaken of response data from the St John Ambulance Service in the Christchurch region from January 2008 to May 2011. Statistical significance was established by Chi-squared testing. 


\section{Results}

After the September 2010 earthquake, overall demand increased by $14 \%$ compared to the same months in 2009. However, in the months after the February 2011 earthquake, a decrease in overall demand from the previous year was observed; the only instance in the entire 2008-2011 period that this phenomenon was observed. Change in demand varied by category of priority response. Response time KPIs were adversely affected by both earthquakes.

\section{Conclusions}

All ambulance providers need disaster plans to ensure continuity of service provision after a major catastrophic event. In Christchurch the demand for, and performance of, emergency ambulances during the recovery period was influenced by factors including evacuation of the population from the affected region and functionality of the infrastructure.

\section{Australian Medical Assistance Teams (AUSMAT) the Paramedic perspective Abigail Trewin and lan Norton}

National Critical Care and Trauma Response Centre, Darwin, Northern Territory, Australia

\section{Background}

AUSMAT (Australian Medical Assistance Team) is a civilian medical team responding to a disaster with the purposes of rendering emergency medical care. The National Critical Care and Trauma Response Centre was established by the Federal Government in response to the 2002/2005 Bali Bombings and 2004 Asian Tsunami. The NCCTRC is funded to provide, a level one AUSMAT, Training and Disaster response equipment under the direction of the Australian Health Protection Committee. The NCCTRC provided the majority of AusMAT personnel to the Pakistan response in 2010. The NCCTRC AusMAT training program includes a broad spectrum of health professionals and provides training to participants from across each state and territory. It prepares responders for the austere environments of a disaster zone, working with limited resources while meeting key SPHERE outcomes.

\section{Objective}

A core focus has been ensuring paramedics and other key emergency services personnel are not overlooked in the selection of a team suitable for response. Creating opportunity for paramedics to be included and recognised for their key attributes is fundamental in ensuring the creation of relevant teams. Ensuring Paramedics understand the key attributes for AusMAT deployment and how to engage in the relevant training is essential in ensuring this health profession is not overlooked.

\section{Methods}

The training has been developed through the recognition of international principles and the guidance from leading world experts and the relevant federal bodies. It has been tailored for a variety of audiences and has now been adopted by National AusMAT State and Territory groups as mandatory training for AusMAT personnel. The course includes, convoy driving, negotiation, security awareness, weapons awareness, controlled road blocks and personal survival. The courses run for a period of 3-4 days and incorporates a "deployment" into the field to ensure candidates "self select' when exposed to reality of austere environments.

\section{The Effectiveness of Airway Management in the Pre Hospital Treatment of Traumatic Brain Injury Joseph Cuthbertson}

\section{St John Ambulance WA Inc, WA, Australia}

\section{Objectives}

There is concern over mortality and the potential for secondary brain injury in the head-injured patient. The use of advanced airway management and rapid sequence intubation (RSI) in the pre hospital treatment of patients with Traumatic Brain Injury (TBI) is controversial. Currently in St John Ambulance Service Australia (WA) Inc. three forms of pre hospital treatment are utilized to manage the airway of the adult head-injured patient. If attended by on-road paramedic staff, basic airway management is utilized unless the patient is unconscious and areflexic, in which case advanced airway management utilizing endotracheal intubation or laryngeal mask insertion is authorised. In the critical care paramedic setting, the severely head-injured patient can be managed utilizing paramedic initiated rapid sequence intubation techniques and on-going sedation, paralysis and ventilation. There is a lack of data evaluating the risk and outcomes involved with these techniques when utilised to treat head-injured patients by paramedics from the Western Australian ambulance service. In this study we provide an updated evaluation of outcomes associated with each procedure.

\section{Methods}

The research framework will be that of a retrospective, observational study of patients transported and treated between January 2004 and January 2009 in Western Australia. As the designated state trauma centre, all major trauma patients are transported to Royal Perth Hospital, where a trauma registry records assessment and treatment details for all admitted patients. All patients admitted to Royal Perth Hospital trauma unit with a head abbreviated injury scale $>3$ 
transported and treated pre hospital by St John Ambulance WA paramedics from January 2004 to January 2009 will be included. Hospital records of patient outcomes will be matched with pre hospital records.

\section{Results \\ Pending \\ Conclusions \\ Pending}

\section{Paramedic graduate destination and internship in New Zealand.}

Paul Davey ${ }^{1}$ and Tim Bradley ${ }^{2}$

${ }^{1}$ AUT University, Auckland, New Zealand

${ }^{2}$ St John, Auckland, New Zealand

\section{Introduction}

Higher education is preparing Paramedic graduates for employment in ambulance services and equipping employed working Paramedics for advances in scope of practice. AUT University and the Order of St John have worked closely to ensure that the degree graduate is road ready. There is nothing so practical as good theory and nothing so theoretically interesting as good practice (Barrows, 1992).

\section{Objectives}

To examine paramedic graduate destination and the internship model in New Zealand.

\section{Methods}

A critical review of the Order of St John internship programme was undertaken using surveys $(n=55)$.

\section{Findings and Conclusions}

The AUT, University degree was established in 2002. Clinical placement hours of a minimum of 1080 hours over three years provide essential links between theory and practice. Advanced life support competencies are embedded in year three of the degree. A four step internship model was designed in 2007 for pre-employment graduates entering the ambulance service. This programme established six monthly goals, workshops, and assessment requirements for increasing scopes of practice. Challenging assessments and applying for increased scope of practice was optional but encouraged. Some graduates displayed potential to progress but lost confidence over time. Attrition of advanced life support knowledge and skill was observed in some graduates due to the optional nature of the programme. New graduates require induction and on going structured support to operate effectively before taking on increased levels of clinical responsibility. The optional element for challenging clinical standards assessment for increased scope for practice should be removed to mitigate attrition of knowledge and skill. The St John Internship Model is am evolving robust method for further developing the talents of graduates.

\section{A Partnership Model for Prehospital Education}

Paul Burke ${ }^{1,2}$, Dianne Inglis ${ }^{1}$, Caitlin Walker ${ }^{1}$, Rod Crole ${ }^{1}$, Jeff Kenneally ${ }^{1}$

${ }^{1}$ Ambulance Victoria, Melbourne, Victoria, Australia

${ }^{2}$ Monash University, Melbourne, Victoria, Australia

\section{Objectives}

This presentation highlights the benefits for establishing a pre-hospital educational program involving partnerships between pre-hospital providers, tertiary institutions, and other healthcare and emergency service providers.

\section{Methods}

An educational program titled Clinical Grand Rounds was established, based on the traditional concept of medical grand rounds, with the collaboration of Ambulance Victoria and a number of external stakeholders from universities and hospitals in Melbourne. The collaboration is in line with the Australian Learning and Teaching Council 2009 report into Paramedic education (1). Evaluation of the program has consisted of questionnaires distributed at each session.

\section{Results}

The evaluation done to date only reflects the first presentation, and was overwhelmingly positive. The respondents indicated on a 4 point scale an average of 3.69 for improved understanding of the topic. The likelihood of attending another Grand Rounds session was rated at an average of 3.90. The cost in time of organising and presenting the session was not calculated. The financial costs were negligible as most resources were provided by the partner organizations in exchange for a variety of benefits from their involvement in the program.

\section{Conclusions}

AV's Clinical Grand Rounds provides a model for a pre-hospital education program which can be implemented at low-cost, and will help to foster understanding and relationships between prehospital providers, hospitals, tertiary institutions and other emergency services. This presentation will discuss the short and long term benefits of the program. 


\section{UK and Victorian, Acute Pain Guidelines Compared}

Scott Stewart ${ }^{1}$ and Paul Burke ${ }^{2}$

${ }^{1}$ Victoria University, Melbourne, Australia

${ }^{2}$ St Georges, University of London, London, UK

\section{Objectives}

To compare the effectiveness of different analgesia guidelines used by two "World Class" ambulance services to suggest optimal treatment regimes.

\section{Methods}

The guidelines for acute pain management of UK Ambulance Service Clinical Practice Guidelines 2006 and Ambulance Victoria, Australia Clinical Guidelines 2010 are presented in summary form. The available outcome measures are then compared

\section{Results}

The management of acute pain by out of hospital providers is one of the highest priorities after reducing morbidity and mortality. Historically oligoanalgesia and under treatment have been widely reported. Under treated pain has many adverse effects including, reduced patient satisfaction, reduced patient compliance and the development of pain syndromes. Recently the guidelines of many ambulance services have been widened to increase the scope of analgesia with improved availability of opiates and novel analgesics

Recently a number of other analgesics have become available to UK ambulance staff, including Paracetamol, Ibuprofen and Morphine Sulphate in addition to Nitrous Oxide. Victoria shares the option of Morphine but has Methoxyflurane, Fentanyl and has trialled Ketamine.

Lack of knowledge has been suggested as a key issue in ineffective pain management. This has been addressed by the increasing move to Higher Education for Paramedic Staff.

Children however are not as well catered for particularly with basic providers and antiemetic availability.

\section{Conclusions}

Adequate education would enable all ambulance teams to have adequate assessment and treatment options available to them. A lack of published data prevents the use of outcomes to evaluate the effectiveness of these regimes. A Combination of the two guidelines would appear to be closer to the optimal solution. This would provide options for managing the diverse problems and situations encountered. Paediatric analgesia is particularly in need of improvement.

\section{Preparedness of International paramedic academics to teach Evidence Based Practice \\ Scott Stewart and Elizabeth Thyer}

Victoria University, Melbourne, Australia

\section{Objectives}

To evaluate the literature regarding preparedness of paramedic academics to teach Evidence Based Practice (EBP).

\section{Methods}

A literature review was undertaken using Medline, OVID, EMBASE and Cochrane Library databases with MeSH subject headings: ambulance paramedic, EMS, EMT, education, Evidence based practice, paramedic, prehospital, and outof-hospital. The literature centred on medicine, psychology, nursing, and allied health, but no literature on preparedness of paramedic academics to teach EBP was found.

\section{Results}

EBP is benchmarked best practice in most health disciplines (Dawes, et al., 2005; De Smedt, Buyl, \& Nyssen, 2006; Tippett, Clark, Woods, \& FitGerald, 2003) and paramedics have also recently embraced EBP (Jensen, Petrie, \& Travers, 2009). Despite an increase in activity in recent years, paramedic research lags behind other health disciplines (Tippett, et al., 2003) which impacts its application to EBP in both Australia and internationally (Bledsoe, 2003; Centre for Prehospital Research, 2008; Sayre, White, Brown 2002).

The move to higher education for paramedics is relatively recent, making appropriate staffing of degrees problematic. Some paramedic academics were not educated at a higher education level and/or have limited research experience (O'Meara, 2006) and this increases the complexity of transitioning into, and developing in, academia. Compounding this is the difficulty in maintaining clinical competency concurrent with teaching and research (Centre for Prehospital Research, 2008; Pointon \& Williams, 2008). The importance of this is highlighted as EBP is seen to be most successful when it relates to real clinical decisions and is integrated with other knowledge, clinical expertise and patient circumstances (Straus, et al., 2011) raising questions of paramedic academic preparedness to teach EBP.

\section{Conclusions}

To competently teach EBP, the paramedic academic needs to understand the EBP process, be able to evaluate the quality of evidence, apply evidence to the out-of-hospital setting and deliver this to students utilising sound pedagogy. 


\section{How effective are first responder programs in Australia? \\ Stephen Burgess and Malcolm Boyle}

Monash University, Frankston, Victoria, Australia

\section{Objectives}

Early access to defibrillation for cardiac arrest patients is highlighted in the seminal paper describing the "chain of survival" concept. The use of fire fighters as "first responders" to acute incidents, especially cardiac arrests, commenced in the mid 1980s in North America. The objective of the study was to identify Australian first responder programs and report their effect on survival rate from cardiac arrest.

\section{Methods}

A literature search was conducted using medical electronic databases, MEDLINE CINHAL, and EMBASE with the databases searched from their beginning until the end of May 2011. References from retrieved articles were reviewed. Keywords included prehospital, out of hospital, first aid, and variations of first responder. Articles were included if they reported on Australian first responder programs and their outcomes, including survival to discharge from hospital. Articles were excluded if they were not written in English and were hospital based.

\section{Results}

There were 430 articles located by the search with seven articles met the inclusion criteria. Two articles were excluded as they did not report survival outcomes. A study at the MCG and Shrine of Remembrance involving St John Ambulance Australia realised $71.4 \%(n=20)$ survivors over five years, the Hatzolah program realised $26 \%(n=5)$ survivors over eight years. A study involving QANTAS aircraft and terminals over five and a half years realised $22 \%(n=6)$ survivors in aircraft and $24 \%(n=4)$ in terminals. In two studies, a pilot study and a 12 month evaluation involving the Metropolitan Fire and Emergency Services Board first responders, there was $4 \%(n=6)$ and $7.2 \%(n=31)$ survivors over two one year periods. Higher survival rates were associated with decreased response times to defibrillation.

\section{Conclusions}

The results of this study suggest the survival from out of hospital cardiac arrest attended by first responders is, in some studies, comparable with international studies.

\section{End-tidal $\mathrm{CO}_{2}$ monitoring via LMA by Advanced Care Paramedics in the out-of- hospital setting \\ Kristi Browne}

The University of the Sunshine Coast, Sunshine Coast, QLD, Australia

\section{Objectives}

The object of this review is to determine whether End-tidal $\mathrm{CO}_{2}$ monitoring $\left(\mathrm{EtCO}_{2}\right)$ would be of benefit for Advanced Care Paramedics through a Laryngeal Mask Airway (LMA). The focus is placed specifically on Queensland, as currently Capnography is only indicated for use in the Queensland Ambulance Service by Intensive Care Paramedics. Whilst Capnography is mandatory for the confirmation of Endotracheal tube placement, it has significantly broader uses which needed to be examined in greater depth.

\section{Methods}

Studies on the benefits and limitations of Capnography (ranging between the years 1997 and 2011) were examined and graded according to the NHMRC guidelines for grading evidence. This analysis specifically focused on the evidence base, risk of bias, consistency, clinical impact, applicability and how generalised the studies were.

\section{Results}

Capnography has consistently predicted return of spontaneous circulation in cardiac arrest, and can also be used to gauge the efficacy of CPR. In cases with major trauma causing poor tissue perfusion \& haemodynamic instability, ventilating according to $\mathrm{EtCO}_{2}$ measurements can lead to hypoventilation, however these changes are predictable and can be accommodated for by targeting $\mathrm{EtCO}_{2}$ levels between $25-30 \mathrm{mmHg}$. In all other cases for patients over $10 \mathrm{~kg}, \mathrm{EtCO}_{2}$ levels correlate to arterial blood gases, and $\mathrm{EtCO}_{2}$ levels between $35-45 \mathrm{mmHg}$ should be targeted to prevent hypoventilation or hyperventilation.

\section{Conclusions}

There is a strong evidence base to support the routine use of Capnography in the out-of-hospital setting with an LMA, particularly in cases of cardiac arrest. $\mathrm{EtCO}_{2}$ monitoring is not a skill which requires extensive training or equipment. Introducing capnography for ACP's on a statewide scale is feasible, and has a low cost to high benefit ratio. 
Gender differences in emergency '000' calls and ambulance response for acute myocardial infarction (AMI) patients. Linda Coventry $^{1}$, Judith Finn ${ }^{1,2}$, Ian Jacobs ${ }^{1,2}$

${ }^{1}$ The University of Western Australia, Perth, Western Australia, Australia

${ }^{2}$ St John Ambulance (Western Australia), Perth, Western Australia, Australia

\section{Objectives}

Minimising time from the onset of symptoms to definitive medical treatment is an important goal for the prehospital management of patients with acute myocardial infarction (AMI). Recognition of the potential AMI during the ' 000 ' call is required to ensure appropriate prioritisation of ambulance response. Our study sought to compare the characteristics of ' 000 ' calls and dispatcher response in men and women with confirmed AMI.

\section{Methods}

Perth Emergency Department discharge data was used to identify AMI patients who arrived by ambulance between Jan 12008 and Oct 312009. The '000' call to St John Ambulance (Western Australia) was transcribed to identify demographic characteristics of the caller, the patient, presenting symptoms and dispatcher priority response. Differences between men and women were analysed using univariable and age-adjusted multivariable techniques. Self-reported time from onset of symptoms to the ' 000 ' call was analysed for a sub-set of patients $(n=861)$.

\section{Results}

During the study period a total of 3,329 AMI patients presented to Perth EDs: 2,100 via ambulance. After pre-defined exclusions, 1,681 emergency ('000') calls were analysed: 621 (36.9\%) of the patients were women. Women were older than men (mean age 77.6 vs 69.1 years, $p<0.001$ ); less likely to report chest pain (54\% vs $69 \%$; OR $=0.54 ; 95 \% \mathrm{Cl} 0.44-0.67$ ) and more likely to report vomiting $(7.4 \%$ vs $4.9 \%$; OR $=1.55 ; 95 \% \mathrm{Cl} 1.03-2.34$ ), even after adjustment for age. Women were less likely than men to be allocated a priority one (lights and sirens) ambulance $(p<0.001)$, even for patients with chest pain. Women delayed longer than men in calling for help (median 104 vs 60 mins, $p=0.006$ ).

\section{Conclusions}

Ambulance dispatch officers (and paramedics) need to be aware of the potential gender differences in AMI presentation, in order to ensure the appropriate prehospital response.

\section{Problem based learning in the Ambulance Service of New South Wales: Xtream2 - Outdoor simulation that supports workplace learning and collaboration in training with front line agencies and university undergraduates. Sharon White}

University of Tasmania, Sydney, Australia

\section{Introduction}

The ASNSW conducts activities that provide points for paramedics to maintain their Certificate to Practice. Participation in Certificate to Practice activities is voluntary and student centred. Xtream2 activities that involve an informal selfreflective approach to clinically focused problem based scenarios. This is supported by a summative assessment. The summative assessment is based on comparisons of the selfreflection by the participant and compared to the summative assessment for the scenario that is completed by the ambulance educator. Collaboration with emergency services and paramedic undergraduates from University of Tasmania has shown successes in building stronger relationships, value adding to training, enhancing the understanding of other roles and breaking down barriers.

\section{Objectives}

Investigate interagency collaboration in training of problem based learning in macro simulation. Participation in the activity and survey was voluntary and not subject to ethics approval and was considered as feedback.

\section{Method}

Surveys were undertaken in order to provide feedback for refinement of the program using an action research model. Completion of the survey was voluntary.

\section{Results}

Paramedics suggested a more formal approach to clinical assessment at the patient's side. State Emergency Service, Rural Fire Service workers were surveyed. Both of these groups agree that collaboration in training would be beneficial to patients and agencies.

\section{Conclusions}

Indicate the paramedics are focused on the development of clinical skills sets in macro simulation and the development of clinical contingencies. Furthermore, paramedics are more likely to agree to assessments when treating patients and they want a more assessment of clinical skills sets in clinical practice settings that are less formal. 
SES and RFS volunteer workers enjoy the participation and collaboration in training, suggesting a breakdown of organisational walls and improving teamwork at scenes. Collaboration in training and assessment for front line agencies and undergraduate students requires investigation from a multi disciplinary research perspective.

\section{From the back of the ambulance to the courtroom: Educating paramedic students in the law \\ Brian Sengstock}

\section{University of the Sunshine Coast, Sippy Downs, Australia}

Traditionally paramedics were trained using an apprenticeship model in the vocational education sector, by State Ambulance Authorities, with a focus on competency based training in clinical skills. In recent times paramedic education has transitioned to the higher education sector as a pre-employment degree qualification. Paramedics are often fearful of the law, adopting the age old analogy that lawyers are "ambulance chasers", rather than seeing the law as an ally to the practice of their profession. The delivery of legal knowledge through lectures and tutorials alone does not allow paramedic students the opportunity to engage with the law in the context of their future profession. An innovative approach to the teaching of law to paramedic students through the use of simulation in conjunction with more traditional approaches to teaching such as lectorials, case discussions and workshops, allows the paramedic students the opportunity to engage not only with their clinical knowledge, but also with the law and legal process.

\section{Despite ambulance services becoming more} aggressive with a variety of new approaches to reducing errors which may result in litigation, evidence based paramedic practice and root cause analysis for unexpected patient outcomes cannot fully alleviate the risk of litigation. Paramedics are human and humans by their very nature are imperfect and make mistakes. Through fully integrating a litigation matter from the service of the statement of claim and defence, through to trial and ultimately judgment, into the course, students are provided with a unique opportunity to participate in the pre-trial and trial elements of a litigation matter. This unique approach allows participants to experience the factual and legal components of a matter, without having to endure the stress, cost and uncertainty of an actual litigation. The simulation culminates with participants engaging in a mock trial.

\section{Are acute stroke patients identified by paramedics transported to appropriate stroke thrombolysis centres?}

Janet Bray ${ }^{1,2}$, Kelly Coughlan ${ }^{3}$, Ian Mosley ${ }^{2}$, Bill Barger $^{1}$, Chris Bladin ${ }^{2,3}$

${ }^{1}$ Ambulance Victoria, Melbourne, Australia

${ }^{2}$ Monash University, Melbourne, Australia

${ }^{3}$ Box Hill Hospital, Melbourne, Australia

\section{Objective}

Current Ambulance Victoria (AV) clinical practice guidelines instruct paramedics to transport patients they identify as acute strokes (< six hours) with positive Melbourne Ambulance Stroke Screen (MASS) criteria to the nearest thrombolysis stroke centres (TSC). The aim of this study is to determine if this practice is followed.

\section{Methods}

Over a six month period, metropolitan and rural cases with a primary assessment of stroke < six hours were examined. Cases not transported to TSCs were reviewed for thrombolysis eligibility and reason for destination.

\section{Results}

For the six month study period, paramedics identified 1451 and 471 cases as acute stroke in metropolitan and rural areas, respectively. The majority of metropolitan cases were transported to TSCs $(n=1285,88 \%)$, but only $65 \%(n=305)$ of rural cases; a further $3 \%(n=47)$ of metropolitan cases and $9 \%(n=43)$ of rural cases were transported to a hospital with a stroke unit, but not offering thrombolysis. However, the majority of acute strokes transported elsewhere were ineligible for thrombolysis; only 24 (of 164) metropolitan cases and 43 (of 166) rural cases were considered eligible from available documentation. Most of these rural cases occurred in the Grampian and Loddon Mallee regions, where transport to the nearest TSC is > 60 minutes. The most common reason for choice of destination in those not transported to a TSC that were MASS positive was "closest public ED" (metropolitan $41 \%$ and rural $65 \%$ ). Other reasons included: closest ED on bypass, clinical guideline, patient or guardian choice, and patient history at destination.

\section{Conclusion}

The majority of acute strokes identified by paramedics in metropolitan regions are transported to stroke thrombolysis centres. Recent developments (commencement of parttime TSCs, telemedicine and "ship \& drip" protocols) in the Grampians and Loddon Mallee regions will improve access to stroke thrombolysis in these areas. 


\section{Determinants of ambulance usage in rural populations during serious health emergencies \\ Buck Reed and Jason Bendall}

Ambulance Research Institute, Sydney, NSW, Australia

\section{Background}

Ambulance utilization rates in high acuity patients have been shown to be lower in rural areas than in urban areas. There is strong anecdotal evidence that rural populations view ambulance services differently than urban users.

Objectives: The objective of this study was to determine the rationale for decision making in rural people who experience serious health emergencies.

\section{Methods}

Results of a previous study into numbers of patients presenting to rural and urban emergency departments was combined with an expansive literature search focusing on both the phenomenon of rural ambulance usage and decision making paradigms employed by those experiencing health emergencies.

\section{Findings}

Adjusted to population sizes, rural people have a lower usage of ambulance in serious medical emergencies than their urban counterparts. Literature suggests that the choice may be due to differences in the way rural people view emergencies and unscheduled events, and their connection to their community. Rural people tend to have a higher degree of self-determination. Their problem solving approaches appear to be more aligned to operating in situations with lower levels of external assistance and they are more likely to be action-oriented. The review indicated that rural populations have a strong sense of being "appropriate users" of ambulance and that they may have a very high standard of what constitutes "appropriate". It was also found that many rural people have a low level of interaction with ambulance and a poor understanding of the capacity of ambulance services to assist in health emergencies.

\section{Conclusions}

Rural people who experience health emergencies make decisions about service access based on a wide range of experiential, cultural and other factors. This may impact upon the likelihood of some rural residents utilising ambulance services at times of serious health emergencies.

\section{Extending Care - Meeting the needs of modern healthcare \\ Jeffrey Andrew}

Ambulance Service NSW, NSW, Australia

\section{Objectives}

The NSW Extended Care Paramedic (ECP) program was developed in collaboration with key stakeholders including NSW Health, Area Health Services, Hospitals, Divisions of General Practice and Ambulance, each contributing to the design of a program aimed at safely reducing Emergency Department presentations for suitable sub acute "000" contacts.

A 10 week intensive education program based at Sydney University, Clinical School for Medicine at Nepean Hospital, with further 12 month clinical and on-going education program to meet the requirements of a qualified Extended Care Paramedic.

The ECP program commenced operations in Sydney West in 2007 with the introduction of first group of 12 ECPs in a proof of concept phase. In 2008 a second course was conducted for a further 5 positions as a regional proof of concept in the Mid North coast of NSW and 5 relief positions. There are now 58 ECP's in NSW.

\section{Methods}

The primary measurable outcome was non transport rate. Other measurable outcomes included patient outcomes, patient satisfaction and external evaluation of cost effectiveness. All patients left at home were followed attempted to be followed up, this had received ethics approval. Questions assessed patient satisfaction; follow up care and any re-presentation to ambulance or hospital.

\section{Results}

As of April 2011, ECP's had responded to 37,000 cases of all priority types. More than 17,000 of these cases requiring no further ambulance resources.

Non-transport rates ranged from $38 \%$ to $60 \%$, compared with $22 \%$ by standard care vehicles A "very satisfied" response was recorded $97 \%$ of call backs.

ED transport avoidance saved an average of $\$ 308$ per patient with additional savings for the wider health system.

\section{Conclusions}

The ECP program in NSW has been demonstrated to be cost effective, safe and have favourable clinical outcomes. It has a high rate of patient satisfaction and contributes to the 
professional development and role diversification for paramedics.

\section{Oxygen for the emergency treatment of acute coronary syndromes - A benefit more presumed than proven \\ Suzanne R Davies ${ }^{1}$ and Paul M Middleton ${ }^{1}$}

${ }^{1}$ Ambulance Research Institute, Ambulance Service of NSW, Sydney, NSW, Australia

${ }^{2}$ Ambulance Research Institute, Ambulance Service of NSW, Sydney, NSW, Australia

\section{Objective}

In 2009, over 90,000 Australians were either hospitalised or died from an acute coronary syndrome (ACS), at a cost of $\$ 18$ billion dollars to the economy. Early, evidence-based emergency care of these patients is critical; approximately one third of deaths will occur in the first hour, one half in the first day. Current prehospital care for patients with suspected ACS aims to maximise the delivery of oxygenated haemoglobin to the myocardium and to slow the pathological process. Treatment consists of salicylic acid, nitrate, morphine and high-flow supplemental oxygen.

\section{Methods}

The authors undertook a review of the literature, to describe and evaluate the current evidence for the routine use of high-flow oxygen in the prehospital management of patients with an ACS. Current international and national prehospital practices and guidelines were also examined.

\section{Result}

No robust evidence for the routine use of untitrated supplemental oxygen in the emergency management of patients with an ACS was identified. The ubiquitous use of supplemental oxygen for ACS has instead been based on two biologically plausible assumptions: (1) that increasing the concentration of oxygen in the blood will also increase the delivery of oxygen to ischaemic myocardium, and (2) that a high concentration of oxygen in the blood (hyperoxia) has no harmful effects. Neither assumption has been supported by clinical evidence.

\section{Conclusion}

There is insufficient evidence to support the routine use of oxygen in the treatment of the normoxic patient suffering an ACS. The use of oxygen for these patients may increase the possibility of an acute myocardial infarct, increase infarct size and possibly increase the risk of mortality. There is a widespread and urgent call for adequately powered, randomised controlled trials investigating the early use of oxygen therapy in ACS, measuring important clinical outcomes and performed in the context of widespread use of reperfusion techniques. 